\title{
Deriving Non-decoupling Effects of Heavy Fields from the Path Integral: a Heavy Higgs Field in an SU(2) Gauge Theory
}

\author{
Stefan Dittmaier ${ }^{1 *}$ and Carsten Grosse-Knetter ${ }^{2 \dagger \dagger}$ \\ ${ }^{1}$ Universität Bielefeld, Fakultät für Physik, \\ Postfach 1001 31, D-33501 Bielefeld, Germany \\ ${ }^{2}$ Université de Montréal, Laboratoire de Physique Nucléaire, \\ C.P. 6128, Montréal, Québec, H3C 3J7, Canada \\ BI-TP 95/01 \\ UdeM-GPP-TH-95-15 \\ hep-ph/9501285 \\ January 1995
}

\begin{abstract}
We describe a method to remove non-decoupling heavy fields from a quantized field theory and to construct a low-energy one-loop effective Lagrangian by integrating out the heavy degrees of freedom in the path integral. We apply this method to the Higgs boson in a spontaneously broken $\mathrm{SU}(2)$ gauge theory (gauged linear $\sigma$ model). In this context, the background-field method is generalized to the non-linear representation of the Higgs sector by applying (a generalization of) the Stueckelberg formalism. The (background) gauge-invariant renormalization is discussed. At one loop the $\log M_{\mathrm{H}}$-terms of the heavy-Higgs limit of this model coincide with the UV-divergent terms of the corresponding gauged non-linear $\sigma$-model, but vertex functions differ in addition by finite (constant) terms in both models. These terms are also derived by our method. Diagrammatic calculations of some vertex functions are presented as consistency check.
\end{abstract}

\footnotetext{
*E-Mail: didi@physik.uni-wuerzburg.de

†E-Mail: knetter@lps.umontreal.ca

$\ddagger$ On leave of absence from the Universität Bielefeld
} 


\section{Introduction}

Effective Lagrangians are used in order to describe the low-energy effects in a theory with heavy particles. The effective Lagrangian contains only light particles and is an approximation to an underlying theory at energy scales much lower than the heavy particles' masses. In general the complete theory is not known and thus the effective Lagrangian contains undetermined parameters. On the other hand, if one knows the underlying theory, the free parameters can be calculated.

There are two different possibilities to construct a low-energy effective Lagrangian of a given theory:

- One may integrate out the heavy particles by diagrammatic methods, i.e. one calculates the contributions of all Feynman diagrams with internal heavy particles to Green functions at a given loop order (usually at one loop) and finds the parameters of the effective theory by matching it to the full theory [1, 2].

- A more fundamental approach is to use functional methods, i.e. to integrate out the heavy particles in the path integral. This generates a functional determinant. The contributions of this determinant to the effective Lagrangian can then be expanded in (inverse) powers of the heavy particles' masses [2, 3, 4, 5, 6].

In the present article we focus on the functional approach. We describe a general and simple method to integrate out non-decoupling heavy fields in the path integral and to obtain a one-loop effective Lagrangian. We explain this method by applying it to the Higgs boson in a spontaneously broken $\mathrm{SU}(2)$ gauge theory, assuming that this boson is very heavy. A large number of articles about functional methods exist, e.g. Refs. [2, 3, 4, 5, 6, . We partially make use of methods developed in some of these works, in particular of those in Ref. [5] and Ref. [6], however, as a whole and in its full detail our procedure has not been described before.

In order to integrate out the heavy fields we use the background-field method (BFM) [7, 8, 9, 10, 11] in which the fields are split into a (classical) background part, which corresponds to tree lines in Feynman graphs, and a quantum part, which corresponds to lines inside loops. Thus, one has to consider only the part in the Lagrangian which is quadratic in the quantum fields in order to construct vertex functions at the one-loop level. The quantum fields associated with the heavy particles can be integrated out by Gaussian integration. The resulting effective Lagrangian still contains the heavy background fields, which can be easily eliminated either diagrammatically by a propagator expansion of the corresponding tree-lines or equivalently by applying the classical equations of motion for the background fields in lowest order.

In most of the existing works not only the heavy quantum fields but all fields are integrated out. Although also in this case an $1 / M$-expansion can formally be done, it will not really be appropriate if not all masses are large. However, an $1 / M$-expansion is a useful tool if $M$ is a heavy particle's mass. Actually, some care has to be taken when integrating out only a part of the quantum fields, viz. one has to diagonalize the Lagrangian, such that the terms which contain both heavy and light quantum fields are removed. This goal can be achieved by appropriately shifting the quantum fields in the path integral [3, 6]. 
A phenomenologically very important field of application for this procedure is the electroweak standard model (SM) provided that the Higgs boson has a mass much larger than the gauge bosons. In this case the Higgs boson can be integrated out and the corresponding low-energy Lagrangian can be constructed. This will be done in a forthcoming paper [12]. In this article we consider a toy model, namely the $\mathrm{SU}(2)$ gauged linear $\sigma$-model (GLSM), which is similar to the SM, but simpler because of the missing $\mathrm{U}(1)$ part and the corresponding mixing in the neutral sector. We integrate out the Higgs boson in this model at the one-loop level and determine the effective Lagrangian to the order $M_{\mathrm{H}}^{0}$ (which includes $\log M_{\mathrm{H}}$ ), i.e. we calculate those terms that contribute in the limit $M_{\mathrm{H}} \rightarrow \infty$. The discussion of this toy model has the advantage, that the physically important and interesting features are essentially the same as in the SM, but the calculations are technically less involved. Therefore, this model is well suited for explaining our method in full detail.

It is well known that the limit of the gauged linear $\sigma$-model for $M_{\mathrm{H}} \rightarrow \infty$ at tree-level is the gauged non-linear $\sigma$-model (GNLSM) [13, 14], which is formally constructed by disregarding the Higgs boson in the non-linear parametrization of the GLSM. At the oneloop level, in Ref. [14] the assumption has been made that the logarithmically divergent contributions to S-matrix elements in the non-renormalizable GNLSM correspond to the logarithmically $M_{\mathrm{H}}$-dependent contributions in the GLSM, provided that the poles in $(D-4)$ - with $D$ being the space-time dimension in dimensional regularization - are appropriately replaced by $\log M_{\mathrm{H}}^{2}$. We find that this is indeed the case, however that there are additional finite and $M_{\mathrm{H}}$-independent differences between the GNLSM and the GLSM at one loop. Thus, the GNLSM with the replacement $2 /(4-D)-\gamma_{E}+\log (4 \pi) \rightarrow$ $\log \left(M_{\mathrm{H}}^{2} / \mu^{2}\right)$ is not identical to the heavy-Higgs limit of the GLSM beyond tree-level.

This result has recently been derived in Ref. [1] for the SM by diagrammatical calculations. Here, we derive it for the $\mathrm{SU}(2)$ model more directly by functional methods. Comparing both methods we find that the functional method has many advantages. E.g. all calculations can be done within the convenient matrix notation, the $1 / M_{\mathrm{H}^{-}}$expansion becomes very straightforward, and the use of the BFM enables us to chose the unitary gauge for the background fields by applying the Stueckelberg formalism [15, 16], which removes the background Goldstone fields from intermediate calculations. Inverting the Stueckelberg transformation at the end, we recover the background Goldstone fields and obtain the result for an arbitrary background gauge.

This article is organized as follows: In Sect. 2 we describe the background-field method and the Stueckelberg formalism for the $\mathrm{SU}(2)$ gauged linear $\sigma$-model and determine the part of the Lagrangian, which contributes to one-loop amplitudes. In Sect. 目 we diagonalize this Lagrangian, i.e. we remove all terms containing both light and heavy quantum fields. In Sect. $⿴$ we integrate out the quantum Higgs field and construct the effective Lagrangian which parametrizes the one-loop effects of the heavy Higgs boson. In Sect. 5 this Lagrangian is written in a manifestly gauge-invariant form. In Sect. 6 we carry out the (gauge-invariant) renormalization. In Sect. (7) the background Higgs field is eliminated, which yields the final effective Lagrangian. In Sect. 8 we check the result of our functional procedure by comparing it with diagrammatical calculations for some vertex functions. Sect. 9 contains the discussion of the result and Sect. 10 the summary. In App. A the explicit form of the Feynman integrals occurring in the calculations are given. 


\section{The Background-Field Method and the Stueckel- berg Formalism}

We consider the Lagrangian of an SU(2) gauged linear $\sigma$-model (GLSM) without fermions. Using the matrix notation the Lagrangian is parametrized by

$$
\mathcal{L}=-\frac{1}{2} \operatorname{tr}\left\{W_{\mu \nu} W^{\mu \nu}\right\}+\frac{1}{2} \operatorname{tr}\left\{\left(D_{\mu} \Phi\right)^{\dagger}\left(D^{\mu} \Phi\right)\right\}+\frac{1}{2} \mu^{2} \operatorname{tr}\left\{\Phi^{\dagger} \Phi\right\}-\frac{1}{16} \lambda\left(\operatorname{tr}\left\{\Phi^{\dagger} \Phi\right\}\right)^{2}
$$

with the gauge fields, field-strength tensors and covariant derivatives given by

$$
\begin{aligned}
W^{\mu} & =\frac{1}{2} W_{i}^{\mu} \tau_{i}, \\
W^{\mu \nu} & =\partial^{\mu} W^{\nu}-\partial^{\nu} W^{\mu}-i g\left[W^{\mu}, W^{\nu}\right], \\
D^{\mu} \Phi & =\left(\partial^{\mu}-i g W^{\mu}\right) \Phi,
\end{aligned}
$$

where $\tau_{i}$ denote the Pauli matrices. The scalar doublet $\Phi$ is linearly parametrized by

$$
\Phi=\frac{1}{\sqrt{2}}((v+H) \mathbf{1}+2 i \varphi)
$$

with

$$
\varphi=\frac{1}{2} \varphi_{i} \tau_{i}, \quad v=2 \sqrt{\frac{\mu^{2}}{\lambda}},
$$

where $v$ is the non-vanishing vacuum expectation value, $H$ is the (physical) Higgs field and the $\varphi_{i}$ are the (unphysical) Goldstone fields. However, in order to construct the heavyHiggs limit of this model, it is more useful to use instead the non-linear parametrization of the scalar sector [16], which is specified by

$$
\Phi=\frac{1}{\sqrt{2}}(v+H) U
$$

where $H$ (unlike in the linear parametrization) is an $\mathrm{SU}(2)$ singlet and the Goldstone fields are arranged to form a unitary matrix $U$. A convenient representation of $U$ is given by the exponential form

$$
U=\exp \left(2 i \frac{\varphi}{v}\right)
$$

The linear parametrization (2.3) und the non-linear one (2.5), (2.6) are physically equivalent, i.e. they yield the same S-matrix, although Green functions are different. The reason for this is that the Jacobian determinant of the field transformation, which relates (2.3) to (2.5), only yields contributions proportional to $\delta^{4}(0)$ [16], which vanish in dimensional regularization. From the GLSM with the parametrization (2.5) the corresponding gauged non-linear $\sigma$-model (GNLSM) 13, 14] can be obtained simply by disregarding the Higgs field rendering the modulus of $\Phi$ constant, $\operatorname{tr}\left\{\Phi^{\dagger} \Phi\right\} \rightarrow v^{2}=$ const. The GNLSM is the $M_{\mathrm{H}} \rightarrow \infty$ limit of the GLSM at tree level [14. It will turn out later in this work that the $M_{\mathrm{H}} \rightarrow \infty$ limit of the GLSM at one loop is the GNLSM plus some effective interaction terms. Therefore, the non-linear parametrization, (2.5) with (2.6), is the more adequate 
one for integrating out the Higgs boson. With this parametrization the Lagrangian (2.1) becomes

$$
\begin{aligned}
\mathcal{L}= & -\frac{1}{2} \operatorname{tr}\left\{W_{\mu \nu} W^{\mu \nu}\right\}+\frac{1}{2}\left(\partial_{\mu} H\right)\left(\partial^{\mu} H\right)+\frac{1}{4}(v+H)^{2} \operatorname{tr}\left\{\left(D_{\mu} U\right)^{\dagger}\left(D^{\mu} U\right)\right\} \\
& +\frac{1}{2} \mu^{2}(v+H)^{2}-\frac{1}{16} \lambda(v+H)^{4},
\end{aligned}
$$

where the Goldstone fields $\varphi_{i}$ occur only in the kinetic term of the scalars owing to the unitarity of $U$.

The Lagrangian (2.7) contains terms cubic and quartic in the Higgs field $H$. Thus, the integral which has to be performed when integrating out $H$ in the path integral is not of Gaussian type. At one loop order this problem can be circumvented by applying the background-field method (BFM) [7, 8, 9, 10, 11], in which the fields are split into (classical) background fields and quantum fields such that the functional integration is only performed over the latter. In this formalism the quantum fields appear only inside loops and the background fields only on tree lines. This means that terms higher than quadratic in the quantum fields only contribute to higher loop orders, but can be neglected in one-loop calculations. Therefore, the Lagrangian to be considered is quadratic in the quantum Higgs field, which can then be integrated out by Gaussian integration.

The BFM for the SM has been formulated in Refs. [9, 10, 11], based on the linear parametrization of the Higgs-Goldstone sector (2.3). For our purpose we have to modify this procedure and to adapt it to the non-linear parametrization (2.5) with (2.6). As usual, we split the gauge and Higgs fields:

$$
W^{\mu} \rightarrow \hat{W}^{\mu}+W^{\mu}, \quad H \rightarrow \hat{H}+H,
$$

where $\hat{W}^{\mu}$ and $\hat{H}$ are the background fields and $W^{\mu}$ and $H$ are the quantum fields. However, for the Goldstone fields (2.6) it is more appropriate to do a non-linear split, namely [6]

$$
U \rightarrow \hat{U} U
$$

where $\hat{U}$ and $U$ are parametrized in terms of the background and quantum Goldstone fields $\hat{\varphi}_{i}$ and $\varphi_{i}$ according to (2.4) and (2.6), respectively.

The advantage of the non-linear split (2.9) is the following: we can now apply the Stueckelberg formalism [15, 16] - or stricly speaking a generalization of this formalism to the BFM - in order to remove the background Goldstone fields $\hat{\varphi}_{i}$ from the Lagrangian. We apply the following transformation to the background and quantum vector fields [6]:

$$
\hat{W}^{\mu} \rightarrow \hat{U} \hat{W}^{\mu} \hat{U}^{\dagger}+\frac{i}{g} \hat{U} \partial^{\mu} \hat{U}^{\dagger}, \quad W^{\mu} \rightarrow \hat{U} W^{\mu} \hat{U}^{\dagger}
$$

One can easily see that the covariant derivatives and field-strength tensors, as defined in (2.2), transform under this Stueckelberg transformation as

$$
D^{\mu} \hat{U} U \rightarrow \hat{U} D^{\mu} U, \quad\left(\hat{W}^{\mu \nu}+W^{\mu \nu}\right) \rightarrow \hat{U}\left(\hat{W}^{\mu \nu}+W^{\mu \nu}\right) \hat{U}^{\dagger} .
$$

Note that the covariant derivative $D^{\mu}$ in (2.11) contains the sum $\hat{W}^{\mu}+W^{\mu}$ of the background and quantum gauge fields. Thus, the effect of the Stueckelberg transformation 
(2.10) is simply to remove the background Goldstone fields from the Lagrangian, i.e. $U \rightarrow \mathbf{1}$, while leaving everything else unaffected.

This means that such a Stueckelberg transformation corresponds to the choice of the unitary gauge (U-gauge) for the background fields. It is an advantage of the BFM that different gauges can be chosen for the background fields and the quantum fields [8, 10, 11]. While a choice of the U-gauge for the quantum fields would complicate loop calculations, the U-gauge for the background fields causes no problems because these fields do not occur in loops. Instead, the background U-gauge reduces the number of terms to be considered in the subsequent treatment (or, equivalently, the number of Feynman diagrams in a diagrammatic procedure). Actually, we will choose a generalized $\mathrm{R}_{\xi}$-gauge for the quantum fields later. By doing the Stueckelberg transformation (2.10) inversely after all calculations have been done, the $\hat{\varphi}_{i}$ can easily be recovered and an arbitrary other gauge for the background fields may be chosen.

The application of the Stueckelberg formalism within the BFM has another important advantage: it automatically ensures the invariance of the effective action under gauge transformations of the background fields. In the conventional formalism, the FaddeevPopov quantization, i.e. the introduction of a gauge-fixing and a ghost term destroys the gauge invariance of the effective action. However, in the BFM it only destroys the invariance with respect to gauge transformations of the quantum fields but a gauge-fixing term for the quantum fields can be chosen such that background gauge invariance is still maintained [8, 9, 10, 11]. This invariance implies then simple Ward identities [8, 10, 11]. In general, the demand of background gauge invariance restricts the choice of the gauge-fixing term. However, after applying the Stueckelberg transformation (2.10) all quantities are automatically background gauge-invariant. This can be seen as follows: apply an SU(2) gauge transformation to the background fields before the Stueckelberg transformation (2.10) is done:

$$
\hat{H} \rightarrow \hat{H}, \quad \hat{U} \rightarrow S \hat{U}, \quad \hat{W}^{\mu} \rightarrow S \hat{W}^{\mu} S^{\dagger}+\frac{i}{g} S \partial^{\mu} S^{\dagger}
$$

with

$$
S=\exp (i g \theta), \quad \text { with } \quad \theta=\frac{1}{2} \theta_{i} \tau_{i}
$$

where $\theta_{i}$ are the group parameters, and transform the quantum fields according to

$$
W^{\mu} \rightarrow S W^{\mu} S^{\dagger}
$$

(2.10) implies that the fields obtained after the Stueckelberg transformation are singlets under (2.12), (2.14). Thus, an arbitrary gauge-fixing term written in terms of these automatically fulfills the requirement of background gauge invariance.

Next, we have to determine that part of the Lagrangian (2.7), which is relevant for oneloop calculations; i.e. the part quadratic in the quantum fields. While the pure background part describes the tree-level effects, the part linear in the quantum fields is irrelevant, and the terms with third or higher powers of the quantum fields contribute only at higher loop orders [7, 8]. Inserting (2.8), (2.9) and (2.10) into (2.7) and defining

$$
\begin{aligned}
\hat{D}_{\varphi}^{\mu} X & =\left(\partial^{\mu}-i g \hat{W}^{\mu}\right) X, \\
\hat{D}_{W}^{\mu} X & =\partial^{\mu} X-i g\left[\hat{W}^{\mu}, X\right],
\end{aligned}
$$


one arrives at

$$
\begin{aligned}
\mathcal{L}^{1-\text { loop }}= & \operatorname{tr}\left\{W_{\mu}\left(g^{\mu \nu} \hat{D}_{W}^{2}-\hat{D}_{W}^{\mu} \hat{D}_{W}^{\nu}+2 i g \hat{W}^{\mu \nu}+g^{\mu \nu} \frac{1}{4} g^{2}(v+\hat{H})^{2}\right) W_{\nu}\right\} \\
& -\operatorname{tr}\left\{\varphi\left(\frac{1}{v^{2}} \hat{D}_{\varphi \mu}(v+\hat{H})^{2} \hat{D}_{\varphi}^{\mu}+g^{2} \frac{1}{v^{2}}(v+\hat{H})^{2} \hat{W}_{\mu} \hat{W}^{\mu}\right) \varphi\right\} \\
& -\frac{1}{2} H\left(\partial^{2}-\mu^{2}+\frac{3}{4} \lambda(v+\hat{H})^{2}-\frac{1}{2} g^{2} \operatorname{tr}\left\{\hat{W}_{\mu} \hat{W}^{\mu}\right\}\right) H \\
& -2 g \frac{1}{v}(v+\hat{H}) H \operatorname{tr}\left\{\hat{W}_{\mu} \partial^{\mu} \varphi\right\} \\
& +g^{2}(v+\hat{H}) H \operatorname{tr}\left\{\hat{W}_{\mu} W^{\mu}\right\} \\
& -g \frac{1}{v}(2 v+\hat{H}) \hat{H} \operatorname{tr}\left\{W_{\mu} \partial^{\mu} \varphi\right\}-2 i g^{2} v \operatorname{tr}\left\{W_{\mu} \hat{W}^{\mu} \varphi\right\}+g v \operatorname{tr}\left\{\varphi \hat{D}_{W}^{\mu} W_{\mu}\right\} \\
& +\mathcal{L}_{\text {gf }}+\mathcal{L}_{\text {ghost }} .
\end{aligned}
$$

Now, we have to fix the gauge of the quantum fields by introducing an appropriate gaugefixing term $\mathcal{L}_{\text {gf }}$. Similar to the procedure in the linear parametrization [9, 10, 11], we choose this term such that the last term in (2.16), which contains a $W \varphi$-mixing, is cancelled. The gauge-fixing term is

$$
\mathcal{L}_{\text {gf }}=-\frac{1}{\xi} \operatorname{tr}\left\{\left(\hat{D}_{W}^{\mu} W_{\mu}+\frac{1}{2} \xi g v \varphi\right)^{2}\right\} .
$$

Recall that $\mathcal{L}_{\text {gf }}$ in $(2.17)$ is written down in the background U-gauge, i.e. for $\hat{U}=\mathbf{1}$; its full form for arbitrary background gauges is obtained by inverting the Stueckelberg transformation (2.10). As mentioned above, the Stueckelberg formalism ensures that this term and the corresponding ghost term are invariant under gauge transformations of the background fields.

The ghost Lagrangian $\mathcal{L}_{\text {ghost }}$, which corresponds to the gauge-fixing term $\mathcal{L}_{\text {gf }}$ (2.17), can be easily derived as usual. Note that the ghosts neither couple to $H$ nor to $\hat{H}$ since the Higgs field, which is an $\mathrm{SU}(2)$ singlet, does not occur in the gauge-fixing term. Therefore, $\mathcal{L}_{\text {gf }}$ and $\mathcal{L}_{\text {ghost }}$ are identical in the GLSM and the GNLSM. Moreover, the ghost term obviously contains in one-loop order (i.e. reduced to its part quadratic in the quantum fields) no other quantum fields then ghosts and remains unaffected by all our manipulations.

Inserting these terms into (2.16) and expressing the parameters $v, \mu^{2}$ and $\lambda$ through the gauge-boson and Higgs masses $M_{\mathrm{W}}$ and $M_{\mathrm{H}}$, respectively,

$$
v=\frac{2 M_{\mathrm{W}}}{g}, \quad \mu^{2}=\frac{1}{2} M_{\mathrm{H}}^{2}, \quad \lambda=\frac{g^{2} M_{\mathrm{H}}^{2}}{2 M_{\mathrm{W}}^{2}},
$$

one finally finds:

$$
\begin{aligned}
\mathcal{L}^{1-\text { loop }}= & \operatorname{tr}\left\{W_{\mu} \Delta_{W}^{\mu \nu} W_{\nu}\right\}-\operatorname{tr}\left\{\varphi \Delta_{\varphi} \varphi\right\}-\frac{1}{2} H \Delta_{H} H \\
& +H \operatorname{tr}\left\{X_{H \varphi} \varphi\right\}+H \operatorname{tr}\left\{X_{H W}^{\mu} W_{\mu}\right\}+\operatorname{tr}\left\{W_{\mu} X_{W \varphi}^{\mu} \varphi\right\}+\mathcal{L}_{\text {ghost }}
\end{aligned}
$$

with

$$
\Delta_{W}^{\mu \nu}=g^{\mu \nu}\left(\hat{D}_{W}^{2}+M_{\mathrm{W}}^{2}\left(1+\frac{\hat{H}}{v}\right)^{2}\right)+\frac{1-\xi}{\xi} \hat{D}_{W}^{\mu} \hat{D}_{W}^{\nu}+2 i g W^{\mu \nu}
$$




$$
\begin{aligned}
\Delta_{\varphi} & =\partial^{\mu}\left(1+\frac{\hat{H}}{v}\right)^{2} \partial_{\mu}+\xi M_{\mathrm{W}}^{2}-2 i g\left(1+\frac{\hat{H}}{v}\right)^{2} \hat{W}^{\mu} \partial_{\mu}, \\
\Delta_{H} & =\partial^{2}+M_{\mathrm{H}}^{2}+\frac{3}{2} M_{\mathrm{H}}^{2} \frac{\hat{H}}{v}\left(2+\frac{\hat{H}}{v}\right)-\frac{1}{2} g^{2} \operatorname{tr}\left\{\hat{W}^{\mu} \hat{W}_{\mu}\right\}, \\
X_{H \varphi} & =-2 g\left(1+\frac{\hat{H}}{v}\right) \hat{W}^{\mu} \partial_{\mu}, \\
X_{H W}^{\mu} & =2 g M_{\mathrm{W}}\left(1+\frac{\hat{H}}{v}\right) \hat{W}^{\mu}, \\
X_{W \varphi}^{\mu} & =-2 M_{\mathrm{W}} \frac{\hat{H}}{v}\left(2+\frac{\hat{H}}{v}\right) \partial^{\mu}-4 i g M_{\mathrm{W}} \hat{W}^{\mu} .
\end{aligned}
$$

\section{Diagonalization of the one-loop Lagrangian}

The Lagrangian (2.19) contains terms linear and quadratic in the quantum Higgs field $H$. Therefore, removing $H$ by doing the integration over this degree of freedom in the path integral results simply in Gaussian integration. However, the presence of terms linear in $H$ would yield effective terms in which inverses of the operators $\Delta_{i}(2.20)$ act on the other quantum fields and which cannot be evaluated easily.

Therefore, before integrating out $H$ we will rewrite the Lagrangian (2.19) such that $H$ appears only quadratically, and thus, after Gaussian integration the operators $\Delta_{i}^{-1}$ only act on the background fields. The terms linear in $H$ can be removed by shifts (which yield unit Jacobian determinants) in the quantum fields [3, 6] as follows: First, we remove the $H \varphi$-term by shifting the $\varphi$ field. The form of the Lagrangian (2.19) suggests a shift:

$$
\varphi \rightarrow \varphi+\frac{1}{2} \hat{\Delta}_{\varphi}^{-1} X_{H \varphi}^{\dagger} H
$$

Note that on the r.h.s. of (3.1) it is not allowed simply to insert the inverse $\Delta_{\varphi}^{-1}$ of $\Delta_{\varphi}$ since the l.h.s. is a linear combination of Pauli matrices while the r.h.s. would not be. On the other hand, we never need the full inverse $\Delta_{\varphi}^{-1}$ - acting on the space of self-adjoint $2 \times 2$ matrices - but only its restriction $\hat{\Delta}_{\varphi}^{-1}$ to the three-dimensional linear subspace spanned by the Pauli matrices $\tau_{i}$. It turns out to be very useful to define the projection operator $P$,

$$
P X=\frac{1}{2} \tau_{i} \operatorname{tr}\left\{\tau_{i} X\right\},
$$

which maps the $\tau$-matrices onto themselves but the unit matrix to zero. In particular, $P$ acts as the identity on $\varphi(2.4)$ and on $W_{\mu}(2.2)$. Splitting $\Delta_{\varphi}$ into a lowest-order part $\Delta_{0}$, which is proportional to the unit matrix, and a remainder $\Pi$,

$$
\Delta_{\varphi}=\Delta_{0}+\Pi
$$

we can explicitly write down $\hat{\Delta}_{\varphi}^{-1}$ in the form of a perturbative series,

$$
\begin{aligned}
\hat{\Delta}_{\varphi}^{-1} & =\Delta_{0}^{-1} P \sum_{n=0}^{\infty}\left(-\Pi \Delta_{0}^{-1} P\right)^{n} \\
& =\Delta_{0}^{-1} P-\Delta_{0}^{-1} P \Pi \Delta_{0}^{-1} P+\Delta_{0}^{-1} P \Pi \Delta_{0}^{-1} P \Pi \Delta_{0}^{-1} P+\cdots
\end{aligned}
$$


With these definitions we obtain

$$
\left(P \Delta_{\varphi} P\right) \hat{\Delta}_{\varphi}^{-1}=\hat{\Delta}_{\varphi}^{-1}\left(P \Delta_{\varphi} P\right)=P .
$$

Note that in the operator equations (3.4) and (3.5) $P$ (which commutes with $\Delta_{0}$ and its inverse) acts on the whole expression right of it. In the following all inverse operators with a hat are defined analogously, i.e. they are restricted to the linear subspace of the $\tau$-matrices.

Shifting $\varphi$ as given in (3.1) with (3.4) yields

$$
\begin{aligned}
\mathcal{L}^{1-\text { loop }}= & \operatorname{tr}\left\{W_{\mu} \Delta_{W}^{\mu \nu} W_{\nu}\right\}-\operatorname{tr}\left\{\varphi \Delta_{\varphi} \varphi\right\}-\frac{1}{2} H \tilde{\Delta}_{H} H \\
& +H \operatorname{tr}\left\{\tilde{X}_{H W}^{\mu} W_{\mu}\right\}+\operatorname{tr}\left\{W_{\mu} X_{W \varphi}^{\mu} \varphi\right\}+\mathcal{L}_{\text {ghost }},
\end{aligned}
$$

with

$$
\begin{aligned}
\tilde{\Delta}_{H} & =\Delta_{H}-\frac{1}{2} \operatorname{tr}\left\{X_{H \varphi} \hat{\Delta}_{\varphi}^{-1} X_{H \varphi}^{\dagger}\right\}, \\
\tilde{X}_{H W}^{\mu} & =X_{H W}^{\mu}+\frac{1}{2} X_{H \varphi} \hat{\Delta}_{\varphi}^{-1} X_{W \varphi}^{\mu \dagger} .
\end{aligned}
$$

If one would now shift the $W$-field in order to remove the $H W$-term, the $W \varphi$-term would generate a new $H \varphi$-term. Thus, we first shift $\varphi$ in order to remove that term,

$$
\varphi \rightarrow \varphi+\frac{1}{2} \hat{\Delta}_{\varphi}^{-1} X_{W \varphi}^{\mu \dagger} W_{\mu},
$$

and $\mathcal{L}^{1-\text { loop }}$ becomes

$$
\mathcal{L}^{1-\text { loop }}=\operatorname{tr}\left\{W_{\mu} \tilde{\Delta}_{W}^{\mu \nu} W_{\nu}\right\}-\operatorname{tr}\left\{\varphi \Delta_{\varphi} \varphi\right\}-\frac{1}{2} H \tilde{\Delta}_{H} H+H \operatorname{tr}\left\{\tilde{X}_{H W}^{\mu} W_{\mu}\right\}+\mathcal{L}_{\text {ghost }}
$$

with

$$
\tilde{\Delta}_{W}^{\mu \nu}=\Delta_{W}^{\mu \nu}+\frac{1}{4} X_{W \varphi}^{\mu} \hat{\Delta}_{\varphi}^{-1} X_{W \varphi}^{\nu \dagger} .
$$

Now, we can remove the $H W$-term by

$$
W^{\mu} \rightarrow W^{\mu}-\frac{1}{2} \hat{\tilde{\Delta}}_{W}^{-1 \mu \nu} \tilde{X}_{H W, \nu} H
$$

which yields

$$
\mathcal{L}^{1-\text { loop }}=\operatorname{tr}\left\{W_{\mu} \tilde{\Delta}_{W}^{\mu \nu} W_{\nu}\right\}-\operatorname{tr}\left\{\varphi \Delta_{\varphi} \varphi\right\}-\frac{1}{2} H \tilde{\tilde{\Delta}}_{H} H+\mathcal{L}_{\text {ghost }}
$$

with

$$
\tilde{\tilde{\Delta}}_{H}=\tilde{\Delta}_{H}+\frac{1}{2} \operatorname{tr}\left\{\tilde{X}_{H W, \mu} \hat{\tilde{\Delta}}_{W}^{-1 \mu \nu} \tilde{X}_{H W, \nu}^{\dagger}\right\} .
$$

Now, all terms linear in $H$ are removed, however, some terms which do not contain $H$, viz. the $W W$ and the $W \varphi$-term are also changed. In order to reconstruct these terms in their original form we do the shift (3.9) inversely and finally find

$$
\mathcal{L}^{1-\text { loop }}=\operatorname{tr}\left\{W_{\mu} \Delta_{W}^{\mu \nu} W_{\nu}\right\}-\operatorname{tr}\left\{\varphi \Delta_{\varphi} \varphi\right\}-\frac{1}{2} H \tilde{\tilde{\Delta}}_{H} H+\operatorname{tr}\left\{W_{\mu} X_{W \varphi}^{\mu} \varphi\right\}+\mathcal{L}_{\text {ghost }} .
$$




\section{Elimination of the Quantum Higgs field and $1 / \mathrm{M}_{\mathrm{H}^{-}}$ Expansion}

Now, we carry out the functional integration over the field $H$ in the path integral, which yields

$$
\begin{aligned}
Z & \propto \int \mathcal{D} H \mathcal{D} W_{i}^{\mu} \mathcal{D} \varphi_{i} \exp \left[i \int d^{4} x \mathcal{L}^{1-\text { loop }}\right] \\
& \propto \int \mathcal{D} W_{i}^{\mu} \mathcal{D} \varphi_{i}\left\{\operatorname{Det}\left(i \tilde{\tilde{\Delta}}_{H} \delta^{(4)}(x-y)\right)\right\}^{-\frac{1}{2}} \exp \left[\left.i \int d^{4} x \mathcal{L}^{1-\text { loop }}\right|_{H=0}\right] \\
& \propto \int \mathcal{D} W_{i}^{\mu} \mathcal{D} \varphi_{i} \exp \left(i S_{\text {eff }}\right) \exp \left[\left.i \int d^{4} x \mathcal{L}^{1-\text { loop }}\right|_{H=0}\right]
\end{aligned}
$$

with

$$
S_{\text {eff }}=\frac{i}{2} \operatorname{Tr}\left\{\log \left(\tilde{\tilde{\Delta}}_{H} \delta^{(4)}(x-y)\right)\right\},
$$

where "Tr" denotes the functional trace, in distinction from the genuine SU(2) trace "tr".

The functional trace and logarithm now have to be evaluated. Many different attempts to perform this evaluation exist in the literature [2, 3, 4, 5, 6]. Our procedure is essentially based on the method developed in Ref. [5]. We write

$$
\begin{aligned}
\tilde{\tilde{\Delta}}_{H}\left(x, \partial_{x}\right) \delta^{(4)}(x-y) & =\int \frac{d^{4} p}{(2 \pi)^{4}} \tilde{\tilde{\Delta}}_{H}\left(x, \partial_{x}\right) \exp \{i p(x-y)\} \\
& =\int \frac{d^{4} p}{(2 \pi)^{4}} \exp \{i p(x-y)\} \tilde{\tilde{\Delta}}_{H}\left(x, \partial_{x}+i p\right) .
\end{aligned}
$$

Now, the trace can be determined:

$$
\operatorname{Tr}\left\{\log \left(\tilde{\tilde{\Delta}}_{H} \delta^{(4)}(x-y)\right)\right\}=\int d^{4} x \int \frac{d^{4} p}{(2 \pi)^{4}} \log \left(\tilde{\tilde{\Delta}}_{H}\left(x, \partial_{x}+i p\right)\right),
$$

and thus (4.2) implies

$$
\mathcal{L}_{\text {eff }}=\frac{i}{2} \int \frac{d^{4} p}{(2 \pi)^{4}} \log \left(\tilde{\tilde{\Delta}}_{H}\left(x, \partial_{x}+i p\right)\right)
$$

$\tilde{\tilde{\Delta}}_{H}\left(x, \partial_{x}+i p\right)$ can be expanded in terms of derivatives,

$$
\tilde{\tilde{\Delta}}_{H}\left(x, \partial_{x}+i p\right)=\sum_{n=0}^{\infty} \frac{(-i)^{n}}{n !}\left[\frac{\partial^{n}}{\partial p_{\mu_{1}} \ldots \partial p_{\mu_{n}}} \tilde{\tilde{\Delta}}_{H}(x, i p)\right] \partial_{\mu_{1}} \ldots \partial_{\mu_{n}},
$$

which will yield an expression like

$$
\tilde{\tilde{\Delta}}_{H}\left(x, \partial_{x}+i p\right)=p^{2}-M_{\mathrm{H}}^{2}+\Pi\left(x, p, \partial_{x}\right) .
$$

Since $\Pi$ commutes with $p^{2}-M_{\mathrm{H}}^{2}$, the logarithm in (4.5) can now easily be expanded (i.e. without having to apply the Baker-Haussdorf formula). Dropping an irrelevant constant, one finds

$$
\log \tilde{\tilde{\Delta}}_{H}\left(x, \partial_{x}+i p\right)=\sum_{n=1}^{\infty} \frac{(-1)^{n+1}}{n}\left(\frac{\Pi}{p^{2}-M_{\mathrm{H}}^{2}}\right)^{n} .
$$


Since we are interested in the heavy-Higgs limit of the GLSM, we only need to consider those terms, which do not vanish for $M_{\mathrm{H}} \rightarrow \infty$, i.e. all terms that are of the order $M_{\mathrm{H}}^{0}$ (which includes $\log M_{\mathrm{H}}$ ) while inverse powers of $M_{\mathrm{H}}$ can be neglected. The procedure described above yields integrals of the type

$$
\int \frac{d^{4} p}{(2 \pi)^{4}} \frac{p_{\mu_{1}} \ldots p_{\mu_{2 k}}}{\left(p^{2}-M_{\mathrm{H}}^{2}\right)^{l}\left(p^{2}-M^{2}\right)^{m}}, \quad \text { with } \quad l>0 \quad \text { and } \quad M^{2}=M_{\mathrm{W}}^{2} \quad \text { or } \quad \xi M_{\mathrm{W}}^{2},
$$

which are explicitly given in App. A. The factors $\left(p^{2}-M_{\mathrm{W}}^{2}\right)^{-1}$ stem from the expansion of the inverse operators $\hat{\Delta}_{\varphi}^{-1}\left(x, \partial_{x}+i p\right)$ and $\hat{\Delta}_{W}^{-1 \mu \nu}\left(x, \partial_{x}+i p\right)$ in $\tilde{\tilde{\Delta}}_{H}\left(x, \partial_{x}+i p\right)$ according to (4.6) and the factors $\left(p^{2}-M_{\mathrm{H}}^{2}\right)^{-1}$ from the expansion of the logarithm (4.8). The integrals (4.9) are $\mathcal{O}\left(M_{\mathrm{H}}^{n}\right)$ with

$$
n=4+2(k-l-m)
$$

if $n \geq 0$, and $\mathcal{O}\left(M_{\mathrm{H}}^{-2}\right)$ or less if $n<0$ (see (A.1)). This means, when expanding $\log \tilde{\tilde{\Delta}}_{H}\left(x, \partial_{x}+i p\right)$, we only have to consider terms of $\mathcal{O}\left(p^{-4}\right)$ or higher powers of $p$. Furthermore, some of the generated terms contain the background Higgs field $\hat{H}$. This will be eliminated later in Sect. 7 by the replacement

$$
\hat{H} \rightarrow \frac{g M_{\mathrm{W}}}{M_{\mathrm{H}}^{2}} \operatorname{tr}\left\{\hat{W}_{\mu} \hat{W}^{\mu}\right\}+\mathcal{O}\left(M_{\mathrm{H}}^{-4}\right) .
$$

This means, each $\hat{H}$ contributes two negative powers of $M_{\mathrm{H}}$. Finally, there is an explicit $M_{\mathrm{H}}$-dependence arising from the couplings of the background Higgs to the quantum Higgs. Therefore, when expanding $\log \tilde{\tilde{\Delta}}_{H}\left(x, \partial_{x}+i p\right)$ we determine the leading powers of $p, \hat{H}$ and $M_{\mathrm{H}}$ for each term generated and introduce an auxiliary parameter $\zeta$, which counts these powers according to

$$
p_{\mu} \rightarrow \zeta, \quad M_{\mathrm{H}} \rightarrow \zeta, \quad \hat{H} \rightarrow \zeta^{-2}
$$

Then, we only have to consider contributions up to $\mathcal{O}\left(\zeta^{-4}\right)$ and can neglect higher negative powers of $\zeta$. In particular this means, that $\tilde{\tilde{\Delta}}_{H}\left(x, \partial_{x}+i p\right)$ only has to be expanded up to $\mathcal{O}\left(\zeta^{-2}\right)$, because each contribution is multiplied with at least one power of $\left(p^{2}-M_{\mathrm{H}}^{2}\right)^{-1}$ in (4.8).

Using (2.20), $\tilde{X}_{H W}^{\mu}$ and $\tilde{\Delta}_{W}^{\mu \nu}$, defined in (3.8) and (3.11), can be expanded as

$$
\begin{aligned}
\tilde{X}_{H W}^{\mu}\left(x, \partial_{x}+i p\right) & =X_{H W}^{\mu}\left(x, \partial_{x}+i p\right)+\mathcal{O}\left(\zeta^{-1}\right), \\
\tilde{\Delta}_{W}^{\mu \nu}\left(x, \partial_{x}+i p\right) & =\Delta_{W}^{\mu \nu}\left(x, \partial_{x}+i p\right)+\mathcal{O}\left(\zeta^{-2}\right), \\
\Rightarrow \quad \hat{\tilde{\Delta}}_{W}^{-1 \mu \nu}\left(x, \partial_{x}+i p\right) & =\hat{\Delta}_{W}^{-1 \mu \nu}\left(x, \partial_{x}+i p\right)+\mathcal{O}\left(\zeta^{-6}\right) .
\end{aligned}
$$

Thus, $\tilde{\tilde{\Delta}}_{H}\left(x, \partial_{x}+i p\right)$ given by (3.7) and (3.14), can be written as

$$
\begin{aligned}
& \tilde{\tilde{\Delta}}_{H}\left(x, \partial_{x}+i p\right)= \\
& \left(\Delta_{H}+\frac{1}{2} \operatorname{tr}\left\{X_{H W, \mu} \hat{\Delta}_{W}^{-1 \mu \nu} X_{H W, \nu}\right\}-\frac{1}{2} \operatorname{tr}\left\{X_{H \varphi} \hat{\Delta}_{\varphi}^{-1} X_{H \varphi}^{\dagger}\right\}\right)\left(x, \partial_{x}+i p\right)+\mathcal{O}\left(\zeta^{-3}\right) .
\end{aligned}
$$


Now, we expand the quantities occurring in (4.16) as far as necessary. The first term is simply

$$
\Delta_{H}\left(x, \partial_{x}+i p\right)=-\left(p^{2}-M_{\mathrm{H}}^{2}\right)+2 i p_{\mu} \partial^{\mu}+\partial^{2}+\frac{3 g M_{\mathrm{H}}^{2}}{2 M_{\mathrm{W}}} \hat{H}+\frac{3 g^{2} M_{\mathrm{H}}^{2}}{8 M_{\mathrm{W}}^{2}} \hat{H}^{2}-\frac{g^{2}}{2} \operatorname{tr}\left\{\hat{W}^{\mu} \hat{W}_{\mu}\right\} .
$$

Also, the second term can be expanded very easily. Since $X_{H W}\left(x, \partial_{x}+i p\right)$ is of $\mathcal{O}\left(\zeta^{0}\right)$, we only need the leading terms of $X_{H W}\left(x, \partial_{x}+i p\right)$ and $\hat{\Delta}_{W}^{-1}\left(x, \partial_{x}+i p\right)$ in (4.16). Eq. (2.20) yields

$$
\begin{aligned}
X_{H W}^{\mu}\left(x, \partial_{x}+i p\right) & =2 g M_{\mathrm{W}} \hat{W}^{\mu}+\mathcal{O}\left(\zeta^{-2}\right), \\
\Delta_{W}^{\mu \nu}\left(x, \partial_{x}+i p\right) & =-g^{\mu \nu}\left(p^{2}-M_{\mathrm{W}}^{2}\right)+\frac{\xi-1}{\xi} p^{\mu} p^{\nu}+\mathcal{O}\left(\zeta^{1}\right), \\
\Rightarrow \hat{\Delta}_{W}^{-1 \mu \nu}\left(x, \partial_{x}+i p\right) & =-\frac{g^{\mu \nu}}{p^{2}-M_{\mathrm{W}}^{2}} P+(1-\xi) \frac{p^{\mu} p^{\nu}}{\left(p^{2}-M_{\mathrm{W}}^{2}\right)\left(p^{2}-\xi M_{\mathrm{W}}^{2}\right)} P+\mathcal{O}\left(\zeta^{-3}\right) \\
& =-\frac{g^{\mu \nu}}{p^{2}-M_{\mathrm{W}}^{2}} P+\frac{p^{\mu} p^{\nu}}{M_{\mathrm{W}}^{2}}\left[\frac{1}{p^{2}-M_{\mathrm{W}}^{2}}-\frac{1}{p^{2}-\xi M_{\mathrm{W}}^{2}}\right] P+\mathcal{O}\left(\zeta^{-3}\right) .
\end{aligned}
$$

Thus, the second term in (4.16) is

$$
\begin{aligned}
\frac{1}{2} \operatorname{tr}\left\{X_{H W \mu} \hat{\Delta}_{W}^{-1 \mu \nu} X_{H W \nu}\right\}\left(x, \partial_{x}+i p\right)= & -2 g^{2} M_{\mathrm{W}}^{2} \frac{1}{p^{2}-M_{\mathrm{W}}^{2}} \operatorname{tr}\left\{\hat{W}_{\mu} \hat{W}^{\mu}\right\} \\
& +2 g^{2}\left[\frac{p_{\mu} p_{\nu}}{p^{2}-M_{\mathrm{W}}^{2}}-\frac{p_{\mu} p_{\nu}}{p^{2}-\xi M_{\mathrm{W}}^{2}}\right] \operatorname{tr}\left\{\hat{W}^{\mu} \hat{W}^{\nu}\right\} \\
& +\mathcal{O}\left(\zeta^{-3}\right) .
\end{aligned}
$$

The operator $P(3.2)$ does not affect the result (4.21) in this order. The expansion of the expressions occurring in the third term in (4.16) is a bit more involved, because the leading term of $X_{H \varphi}\left(x, \partial_{x}+i p\right)$ is $\mathcal{O}\left(\zeta^{1}\right)$ and thus also some non-leading parts of $X_{H \varphi}\left(x, \partial_{x}+i p\right)$ and $\hat{\Delta}_{\varphi}^{-1}\left(x, \partial_{x}+i p\right)$ have to be taken into account:

$$
\begin{aligned}
X_{H \varphi}\left(x, \partial_{x}+i p\right)= & -2 i g \hat{W}_{\mu} p^{\mu}-2 g \hat{W}_{\mu} \partial^{\mu}-i \frac{g^{2}}{M_{\mathrm{W}}} \hat{H} \hat{W}_{\mu} p^{\mu}+\mathcal{O}\left(\zeta^{-2}\right) \\
\Delta_{\varphi}\left(x, \partial_{x}+i p\right)= & -\left(p^{2}-\xi M_{\mathrm{W}}^{2}\right)+2 i p_{\mu} \partial^{\mu}+\partial^{2} \\
& +2 g \hat{W}_{\mu} p^{\mu}-2 i g \hat{W}_{\mu} \partial^{\mu}-\frac{g}{M_{\mathrm{W}}} \hat{H} p^{2}+\mathcal{O}\left(\zeta^{-1}\right) \\
\Rightarrow \hat{\Delta}_{\varphi}^{-1}\left(x, \partial_{x}+i p\right)= & -\frac{1}{p^{2}-\xi M_{\mathrm{W}}^{2}} P \\
& -\frac{1}{\left(p^{2}-\xi M_{\mathrm{W}}^{2}\right)^{2}} P\left[2 i p_{\mu} \partial^{\mu}+\partial^{2}+2 g \hat{W}_{\mu} p^{\mu}-2 i g \hat{W}_{\mu} \partial^{\mu}-\frac{g}{M_{\mathrm{W}}} \hat{H} p^{2}\right] P \\
& -\frac{4 p_{\mu} p_{\nu}}{\left(p^{2}-\xi M_{\mathrm{W}}^{2}\right)^{3}} P\left[-\partial^{\mu} \partial^{\nu}+i g\left(\partial^{\mu} \hat{W}^{\nu}+\hat{W}^{\mu} \partial^{\nu}\right)+g^{2} \hat{W}^{\mu} P \hat{W}^{\nu}\right] P \\
& +\mathcal{O}\left(\zeta^{-5}\right) .
\end{aligned}
$$

Since $P(3.2)$ acts as the identity as long as there is exactly one Pauli matrix left or right of it and since $X_{H \varphi}\left(x, \partial_{x}+i p\right)$ is a linear combination of the $\tau_{i}$, the $P$ in (4.24) can be 
dropped everywhere except for the very last term contributing to $\hat{\Delta}_{\varphi}^{-1}\left(x, \partial_{x}+i p\right)$, where two Pauli Matrices occur. The third term in (4.16) becomes then

$$
\begin{aligned}
& -\frac{1}{2} \operatorname{tr}\left\{X_{H \varphi} \hat{\Delta}_{\varphi}^{-1} X_{H \varphi}^{\dagger}\right\}\left(x, \partial_{x}+i p\right)= \\
& \frac{1}{p^{2}-\xi M_{\mathrm{W}}^{2}} 2 g^{2}\left[\operatorname{tr}\left\{\left(\partial_{\mu} \hat{W}^{\mu}\right)^{2}\right\}+p_{\mu} p_{\nu} \operatorname{tr}\left\{\hat{W}^{\mu} \hat{W}^{\nu}\right\}\right] \\
& -\frac{p_{\mu} p_{\nu}}{\left(p^{2}-\xi M_{\mathrm{W}}^{2}\right)^{2}} 2 g^{2}\left[\operatorname{tr}\left\{\left(\partial_{\rho} \hat{W}^{\mu}\right)\left(\partial^{\rho} \hat{W}^{\nu}\right)\right\}+4 \operatorname{tr}\left\{\left(\partial^{\mu} \hat{W}^{\nu}\right)\left(\partial_{\rho} \hat{W}^{\rho}\right)\right\}\right. \\
& \left.\quad-2 i g \operatorname{tr}\left\{\left(\partial_{\rho} \hat{W}^{\nu}\right) \hat{W}^{\rho} \hat{W}^{\mu}\right\}\right] \\
& +\frac{p_{\mu} p_{\nu} p_{\rho} p_{\sigma}}{\left(p^{2}-\xi M_{\mathrm{W}}^{2}\right)^{3}} 8 g^{2} \operatorname{tr}\left\{\left(\partial^{\mu} \hat{W}^{\nu}\right)\left(\partial^{\rho} \hat{W}^{\sigma}\right)\right\} \\
& +\mathcal{O}\left(\zeta^{-3}\right) .
\end{aligned}
$$

In determining (4.25), we have already carried out some simplifications, which are strictly speaking only justified in the full expansion of (4.5). More precisely, we have dropped total derivative terms of $\mathcal{O}\left(\zeta^{-2}\right)$, which contribute only to the linear term $(n=1)$ in the expansion of the logarithm (4.8). Such terms yield total derivatives of $\mathcal{L}_{\text {eff }}$ either. Furthermore, we made use of the fact that in all expressions the Lorentz indices can be arbitrarily exchanged. In particular, this and the definition of $P(3.2)$ imply that the only contribution with this operator, which has the form $p_{\mu} p_{\nu} p_{\rho} p_{\sigma} \operatorname{tr}\left\{\hat{W}^{\mu} \hat{W}^{\nu} P \hat{W}^{\rho} \hat{W}^{\sigma}\right\}$, vanishes. Finally, the contributions from the $\hat{H}$ terms in $X_{H \varphi}\left(x, \partial_{x}+i p\right)(4.22)$ and in $\hat{\Delta}_{\varphi}^{-1}\left(x, \partial_{x}+i p\right)$ (4.24) cancel at this order, because $p_{\mu} p_{\nu} p^{2}\left(p^{2}-M_{\mathrm{W}}^{2}\right)^{-2}=p_{\mu} p_{\nu}\left(p^{2}-M_{\mathrm{W}}^{2}\right)^{-1}+\mathcal{O}\left(\zeta^{-2}\right)$. Summing (4.17), (4.21) and (4.25) we find the expansion for (4.16).

Next, we expand $\log \tilde{\tilde{\Delta}}_{H}\left(x, \partial_{x}+i p\right)$ as in (4.8) and integrate over $p$ in order to obtain the effective Lagrangian (4.5). Only the first two terms in the expansion of $\log \tilde{\tilde{\Delta}}_{H}\left(x, \partial_{x}+\right.$ ip) yield $\mathcal{O}\left(\zeta^{-4}\right)$ (or higher) contributions. Furthermore, only the $\hat{H}$-term in (4.17), the $\operatorname{tr}\left\{\hat{W}_{\mu} \hat{W}^{\mu}\right\}$-term in (4.17), and the $p_{\mu} p_{\nu} \operatorname{tr}\left\{\hat{W}^{\mu} \hat{W}^{\nu}\right\}$-term in (4.25) have to be considered in the quadratic part of the expansion of the logarithm. The integrals which have to be calculated are symmetric with respect to exchange of Lorentz indices. We use the notation

$$
I_{k l m}(\xi) g_{\mu_{1} \ldots \mu_{2 k}}=\frac{(2 \pi \mu)^{4-D}}{i \pi^{2}} \int d^{D} p \frac{p_{\mu_{1}} \ldots p_{\mu_{2 k}}}{\left(p^{2}-M_{\mathrm{H}}^{2}\right)^{l}\left(p^{2}-\xi M_{\mathrm{W}}^{2}\right)^{m}},
$$

with $g_{\mu_{1} \ldots \mu_{2 k}}$ being the totally symmetric tensor built of $g_{\mu \nu}$ 's with rank $2 k$. As indicated in (4.26), we use dimensional regularization in order to regularize occurring UV divergencies. $D$ denotes the space-time dimension, and $\mu$ an arbitrary reference mass scale. The explicit expressions for the occurring integrals are given in App. A. Dropping total derivatives, contracting the Lorentz indices and collecting all terms we find: ${ }^{1}$

$$
\mathcal{L}_{\text {eff }}=\frac{1}{16 \pi^{2}} g \frac{3 M_{\mathrm{H}}^{2}}{4 M_{\mathrm{W}}^{2}} I_{010} \hat{H}
$$

\footnotetext{
${ }^{1}$ Note that the neglected remainder in $(4.27)$ is $\mathcal{O}\left(\zeta^{-2}\right)$ and not only $\mathcal{O}\left(\zeta^{-1}\right)$, since integrals like 4.26 vanish for an odd number of $p_{\mu}$ 's.
} 


$$
\begin{aligned}
& +\frac{1}{16 \pi^{2}} g^{2}\left(\frac{3 M_{\mathrm{H}}^{2}}{16 M_{\mathrm{W}}^{2}} I_{010}+\frac{9 M_{\mathrm{H}}^{4}}{16 M_{\mathrm{W}}^{2}} I_{020}\right) \hat{H}^{2} \\
& +\frac{1}{16 \pi^{2}} g^{3}\left(-\frac{3 M_{\mathrm{H}}^{2}}{8 M_{\mathrm{W}}} I_{020}+\frac{3 M_{\mathrm{H}}^{2}}{2 M_{\mathrm{W}}} I_{121}(\xi)\right) \hat{H} \operatorname{tr}\left\{\hat{W}_{\mu} \hat{W}^{\mu}\right\} \\
& +\frac{1}{16 \pi^{2}} g^{2}\left(-\frac{1}{4} I_{010}-M_{\mathrm{W}}^{2} I_{011}(1)+I_{111}(1)\right) \operatorname{tr}\left\{\hat{W}_{\mu} \hat{W}^{\mu}\right\} \\
& +\frac{1}{16 \pi^{2}} g^{2}\left(-I_{112}(\xi)+4 I_{213}(\xi)\right) \operatorname{tr}\left\{\left(\partial_{\mu} \hat{W}_{\nu}\right)\left(\partial^{\mu} \hat{W}^{\nu}\right)\right\} \\
& +\frac{1}{16 \pi^{2}} g^{2}\left(I_{011}(\xi)-4 I_{112}(\xi)+8 I_{213}(\xi)\right) \operatorname{tr}\left\{\left(\partial_{\mu} \hat{W}^{\mu}\right)^{2}\right\} \\
& +\frac{1}{16 \pi^{2}} 2 i g^{3} I_{112}(\xi) \operatorname{tr}\left\{\left(\partial_{\mu} \hat{W}_{\nu}\right) \hat{W}^{\mu} \hat{W}^{\nu}\right\} \\
& +\frac{1}{16 \pi^{2}} 2 g^{4} I_{222}(\xi) \operatorname{tr}\left\{\hat{W}_{\mu} \hat{W}_{\nu}\right\} \operatorname{tr}\left\{\hat{W}^{\mu} \hat{W}^{\nu}\right\} \\
& +\frac{1}{16 \pi^{2}} g^{4}\left(\frac{1}{16} I_{020}-\frac{1}{2} I_{121}(\xi)+I_{222}(\xi)\right)\left(\operatorname{tr}\left\{\hat{W}_{\mu} \hat{W}^{\mu}\right\}\right)^{2} \\
& +\mathcal{O}\left(\zeta^{-2}\right) .
\end{aligned}
$$

\section{Inverting the Stueckelberg transformation}

Now, we write these terms in a more convenient form by introducing non-Abelian fieldstrength tensors (2.2). We first write

$$
\begin{aligned}
\operatorname{tr}\left\{\left(\partial_{\mu} \hat{W}_{\nu}\right)\left(\partial^{\mu} \hat{W}^{\nu}\right)\right\}= & \frac{1}{2} \operatorname{tr}\left\{\hat{W}_{\mu \nu} \hat{W}^{\mu \nu}\right\}+\operatorname{tr}\left\{\left(\partial_{\mu} \hat{W}^{\mu}\right)^{2}\right\} \\
& +i g \operatorname{tr}\left\{\hat{W}_{\mu \nu}\left[\hat{W}^{\mu}, \hat{W}^{\nu}\right]\right\}-\frac{1}{2} g^{2} \operatorname{tr}\left\{\left[\hat{W}_{\mu}, \hat{W}_{\nu}\right]\left[\hat{W}^{\mu}, \hat{W}^{\nu}\right]\right\}, \\
\operatorname{tr}\left\{\left(\partial_{\mu} \hat{W}_{\nu}\right) \hat{W}^{\mu} \hat{W}^{\nu}\right\}= & \frac{1}{4} \operatorname{tr}\left\{\hat{W}_{\mu \nu}\left[\hat{W}^{\mu}, \hat{W}^{\nu}\right]\right\}+\frac{i}{4} g \operatorname{tr}\left\{\left[\hat{W}_{\mu}, \hat{W}_{\nu}\right]\left[\hat{W}^{\mu}, \hat{W}^{\nu}\right]\right\} .
\end{aligned}
$$

The generated traces of four Pauli matrices can be expressed through traces of two $\tau$ 's by using the identity

$$
\operatorname{tr}\left\{\tau_{i} \tau_{j} \tau_{k} \tau_{l}\right\}=\frac{1}{2}\left(\operatorname{tr}\left\{\tau_{i} \tau_{j}\right\} \operatorname{tr}\left\{\tau_{k} \tau_{l}\right\}-\operatorname{tr}\left\{\tau_{i} \tau_{k}\right\} \operatorname{tr}\left\{\tau_{j} \tau_{l}\right\}+\operatorname{tr}\left\{\tau_{i} \tau_{l}\right\} \operatorname{tr}\left\{\tau_{j} \tau_{k}\right\}\right),
$$

which yields

$$
\begin{aligned}
\operatorname{tr}\left\{\left[\hat{W}_{\mu}, \hat{W}_{\nu}\right]\left[\hat{W}^{\mu}, \hat{W}^{\nu}\right]\right\} & =2 \operatorname{tr}\left\{\hat{W}_{\mu} \hat{W}_{\nu} \hat{W}^{\mu} \hat{W}^{\nu}\right\}-2 \operatorname{tr}\left\{\hat{W}_{\mu} \hat{W}^{\mu} \hat{W}_{\nu} \hat{W}^{\nu}\right\} \\
& =2 \operatorname{tr}\left\{\hat{W}_{\mu} \hat{W}_{\nu}\right\} \operatorname{tr}\left\{\hat{W}^{\mu} \hat{W}^{\nu}\right\}-2\left(\operatorname{tr}\left\{\hat{W}_{\mu} \hat{W}^{\mu}\right\}\right)^{2} .
\end{aligned}
$$

Inserting (5.1) and (5.3) into (4.27) we find

$$
\mathcal{L}_{\text {eff }}=\frac{1}{16 \pi^{2}} g \frac{3 M_{\mathrm{H}}^{2}}{4 M_{\mathrm{W}}^{2}} I_{010} \hat{H}
$$




$$
\begin{aligned}
& +\frac{1}{16 \pi^{2}} g^{2}\left(\frac{3 M_{\mathrm{H}}^{2}}{16 M_{\mathrm{W}}^{2}} I_{010}+\frac{9 M_{\mathrm{H}}^{4}}{16 M_{\mathrm{W}}^{2}} I_{020}\right) \hat{H}^{2} \\
& +\frac{1}{16 \pi^{2}} g^{3}\left(-\frac{3 M_{\mathrm{H}}^{2}}{8 M_{\mathrm{W}}} I_{020}+\frac{3 M_{\mathrm{H}}^{2}}{2 M_{\mathrm{W}}} I_{121}(\xi)\right) \hat{H} \operatorname{tr}\left\{\hat{W}_{\mu} \hat{W}^{\mu}\right\} \\
& +\frac{1}{16 \pi^{2}} g^{2}\left(-\frac{1}{4} I_{010}-M_{\mathrm{W}}^{2} I_{011}(1)+I_{111}(1)\right) \operatorname{tr}\left\{\hat{W}_{\mu} \hat{W}^{\mu}\right\} \\
& +\frac{1}{16 \pi^{2}} g^{2}\left(-\frac{1}{2} I_{112}(\xi)+2 I_{213}(\xi)\right) \operatorname{tr}\left\{\hat{W}_{\mu \nu} \hat{W}^{\mu \nu}\right\} \\
& +\frac{1}{16 \pi^{2}} g^{2}\left(I_{011}(\xi)-5 I_{112}(\xi)+12 I_{213}(\xi)\right) \operatorname{tr}\left\{\left(\partial_{\mu} \hat{W}^{\mu}\right)^{2}\right\} \\
& +\frac{1}{16 \pi^{2}} i g^{3}\left(-\frac{1}{2} I_{112}(\xi)+4 I_{213}(\xi)\right) \operatorname{tr}\left\{\hat{W}_{\mu \nu}\left[\hat{W}^{\mu}, \hat{W}^{\nu}\right]\right\} \\
& +\frac{1}{16 \pi^{2}} g^{4}\left(-4 I_{213}(\xi)+2 I_{222}(\xi)\right) \operatorname{tr}\left\{\hat{W}_{\mu} \hat{W}_{\nu}\right\} \operatorname{tr}\left\{\hat{W}^{\mu} \hat{W}^{\nu}\right\} \\
& +\frac{1}{16 \pi^{2}} g^{4}\left(\frac{1}{16} I_{020}-\frac{1}{2} I_{121}(\xi)+4 I_{213}(\xi)+I_{222}(\xi)\right)\left(\operatorname{tr}\left\{\hat{W}_{\mu} \hat{W}^{\mu}\right\}\right)^{2} \\
& +\mathcal{O}\left(\zeta^{-2}\right) .
\end{aligned}
$$

Then, we reintroduce the background Goldstone fields $\hat{\varphi}_{i}$ by inverting the Stueckelberg transformation (2.10), i.e. we transform the background and quantum vector fields as

$$
\hat{W}^{\mu} \rightarrow \hat{U}^{\dagger} \hat{W}^{\mu} \hat{U}+\frac{i}{g} \hat{U}^{\dagger} \partial^{\mu} \hat{U}=\frac{i}{g} \hat{U}^{\dagger} \hat{D}_{\varphi}^{\mu} \hat{U}, \quad W^{\mu} \rightarrow \hat{U}^{\dagger} W^{\mu} \hat{U}
$$

with $\hat{D}_{\varphi}^{\mu}$ being defined in (2.15). Obviously, the transformation of the quantum fields is only needed in the remaining term $\left.\mathcal{L}^{1-\text { loop }}\right|_{H=0}$ in the path integral $(4.1)$, while $\mathcal{L}_{\text {eff }}(5.4)$ only consists of background fields. According to Refs. [1, 14, we introduce a shorthand notation for the covariant derivative

$$
\hat{V}^{\mu}=\left(\hat{D}_{\varphi}^{\mu} \hat{U}\right) \hat{U}^{\dagger}
$$

The transformations of the vector fields, field-strength tensors and derivatives in (5.4) under (5.5) can be written as

$$
\begin{aligned}
\hat{W}^{\mu} & \rightarrow \frac{i}{g} \hat{U}^{\dagger} \hat{V}^{\mu} \hat{U} \\
\hat{W}^{\mu \nu} & \rightarrow \hat{U}^{\dagger} \hat{W}^{\mu \nu} \hat{U} \\
\partial^{\mu} \hat{W}_{\mu} & \rightarrow \frac{i}{g} \partial^{\mu}\left(\hat{U}^{\dagger} \hat{V}_{\mu} \hat{U}\right)=\frac{i}{g} \hat{U}^{\dagger}\left(\hat{D}_{W}^{\mu} \hat{V}_{\mu}\right) \hat{U},
\end{aligned}
$$

with $\hat{D}_{W}^{\mu}$ being defined in (2.15). Applying this to (5.4), we finally find the effective Lagrangian

$$
\begin{aligned}
\mathcal{L}_{\text {eff }}= & \frac{1}{16 \pi^{2}} g \frac{3 M_{\mathrm{H}}^{2}}{4 M_{\mathrm{W}}^{2}} I_{010} \hat{H} \\
+ & \frac{1}{16 \pi^{2}} g^{2}\left(\frac{3 M_{\mathrm{H}}^{2}}{16 M_{\mathrm{W}}^{2}} I_{010}+\frac{9 M_{\mathrm{H}}^{4}}{16 M_{\mathrm{W}}^{2}} I_{020}\right) \hat{H}^{2}
\end{aligned}
$$




$$
\begin{aligned}
& +\frac{1}{16 \pi^{2}} g\left(\frac{3 M_{\mathrm{H}}^{2}}{8 M_{\mathrm{W}}} I_{020}-\frac{3 M_{\mathrm{H}}^{2}}{2 M_{\mathrm{W}}} I_{121}(\xi)\right) \hat{H} \operatorname{tr}\left\{\hat{V}_{\mu} \hat{V}^{\mu}\right\} \\
& +\frac{1}{16 \pi^{2}}\left(\frac{1}{4} I_{010}+M_{\mathrm{W}}^{2} I_{011}(1)-I_{111}(1)\right) \operatorname{tr}\left\{\hat{V}_{\mu} \hat{V}^{\mu}\right\} \\
& +\frac{1}{16 \pi^{2}} g^{2}\left(-\frac{1}{2} I_{112}(\xi)+2 I_{213}(\xi)\right) \operatorname{tr}\left\{\hat{W}_{\mu \nu} \hat{W}^{\mu \nu}\right\} \\
& +\frac{1}{16 \pi^{2}}\left(-I_{011}(\xi)+5 I_{112}(\xi)-12 I_{213}(\xi)\right) \operatorname{tr}\left\{\left(\hat{D}_{W}^{\mu} \hat{V}_{\mu}\right)^{2}\right\} \\
& +\frac{1}{16 \pi^{2}} i g\left(\frac{1}{2} I_{112}(\xi)-4 I_{213}(\xi)\right) \operatorname{tr}\left\{\hat{W}_{\mu \nu}\left[\hat{V}^{\mu}, \hat{V}^{\nu}\right]\right\} \\
& +\frac{1}{16 \pi^{2}}\left(-4 I_{213}(\xi)+2 I_{222}(\xi)\right) \operatorname{tr}\left\{\hat{V}_{\mu} \hat{V}_{\nu}\right\} \operatorname{tr}\left\{\hat{V}^{\mu} \hat{V}^{\nu}\right\} \\
& +\frac{1}{16 \pi^{2}}\left(\frac{1}{16} I_{020}-\frac{1}{2} I_{121}(\xi)+4 I_{213}(\xi)+I_{222}(\xi)\right)\left(\operatorname{tr}\left\{\hat{V}_{\mu} \hat{V}^{\mu}\right\}\right)^{2} \\
& +\mathcal{O}\left(\zeta^{-2}\right),
\end{aligned}
$$

which is manifestly invariant under gauge transformations of the background fields (2.12), because $\hat{W}^{\mu \nu}, \hat{V}^{\mu}$ and $\hat{D}_{W}^{\mu} \hat{V}_{\mu}$ transform covariantly under (2.12), i.e.

$$
\hat{W}^{\mu \nu} \rightarrow S \hat{W}^{\mu \nu} S^{\dagger}, \quad \hat{V}^{\mu} \rightarrow S \hat{V}^{\mu} S^{\dagger}, \quad \hat{D}_{W}^{\mu} \hat{V}_{\mu} \rightarrow S\left(\hat{D}_{W}^{\mu} \hat{V}_{\mu}\right) S^{\dagger}
$$

In this Lagrangian the gauge for the background fields can now be fixed arbitraily, e.g. in the $R_{\xi}$-gauge.

\section{Renormalization}

In the previous sections we dealt with bare parameters and bare fields only. Now, we apply the following renormalization transformation to the parameters

$$
\begin{aligned}
g & \rightarrow g_{0}=g+\delta g, \\
M_{\mathrm{W}}^{2} & \rightarrow M_{\mathrm{W}, 0}^{2}=M_{\mathrm{W}}^{2}+\delta M_{\mathrm{W}}^{2}, \\
M_{\mathrm{H}}^{2} & \rightarrow M_{\mathrm{H}, 0}^{2}=M_{\mathrm{H}}^{2}+\delta M_{\mathrm{H}}^{2}, \\
t & \rightarrow t_{0}=t+\delta t,
\end{aligned}
$$

where bare quantities are marked by a subscript " 0 " in the following. The tadpole term $t=v\left(\mu^{2}-\lambda v^{2} / 4\right)$ is introduced via the term $t \hat{H}(x)$ in the Lagrangian (2.7).2 Consequently, the parameter counterterms are generated by the replacements

$$
\begin{aligned}
g & \rightarrow g_{0}=g+\delta g, \\
v & \rightarrow v_{0}=v+\delta v, \\
\mu^{2} & \rightarrow \mu_{0}^{2}=\mu^{2}+\delta \mu^{2}, \\
\lambda & \rightarrow \lambda_{0}=\lambda+\delta \lambda,
\end{aligned}
$$

\footnotetext{
${ }^{2}$ Strictly speaking, the relations between the parameters given in (2.4) and (2.18) hold for renormalized quantities. We should have taken a non-vanishing tadpole term $t$ into account for the unrenormalized parameters. Instead, we omitted $t$ there in order to avoid confusion, but reintroduce it here.
} 
in the Lagrangian (2.7), where

$$
\begin{aligned}
\frac{\delta v}{v} & =\frac{1}{2} \frac{\delta M_{\mathrm{W}}^{2}}{M_{\mathrm{W}}^{2}}-\frac{\delta g}{g}, \\
\frac{\delta \mu^{2}}{\mu^{2}} & =\frac{\delta M_{\mathrm{H}}^{2}}{M_{\mathrm{H}}^{2}}+\frac{3 g}{2 M_{\mathrm{W}} M_{\mathrm{H}}^{2}} \delta t, \\
\frac{\delta \lambda}{\lambda} & =\frac{\delta M_{\mathrm{H}}^{2}}{M_{\mathrm{H}}^{2}}-\frac{\delta M_{\mathrm{W}}^{2}}{M_{\mathrm{W}}^{2}}+2 \frac{\delta g}{g}+\frac{g}{2 M_{\mathrm{W}} M_{\mathrm{H}}^{2}} \delta t .
\end{aligned}
$$

The masses $M_{\mathrm{W}}$ and $M_{\mathrm{H}}$ are fixed within the on-shell renormalization scheme, which yields the usual conditions (see e.g. Refs. [17, 18])

$$
\begin{aligned}
\delta M_{\mathrm{W}}^{2} & =\operatorname{Re}\left\{\Sigma_{\mathrm{T}}^{\hat{W} \hat{W}}\left(M_{\mathrm{W}}^{2}\right)\right\}, \\
\delta M_{\mathrm{H}}^{2} & =\operatorname{Re}\left\{\Sigma^{\hat{H} \hat{H}}\left(M_{\mathrm{H}}^{2}\right)\right\},
\end{aligned}
$$

where $\Sigma_{\mathrm{T}}^{\hat{W}} \hat{W}$ and $\Sigma^{\hat{H} \hat{H}}$ represent the transversal part of the unrenormalized $\hat{W}$ self-energy and the unrenormalized $\hat{H}$ self-energy, respectively. Concerning the notation of selfenergies, vertex functions etc. we follow Refs. [10, 11] throughout. The tadpole counterterm is defined such that the renormalized tadpole vanishes,

$$
\delta t=-T^{\hat{H}}
$$

In the following it turns out that the renormalization condition for the coupling $g$ does not need to be explicitly specified except for the requirement that it must be defined at a low-energy scale $q^{2}$, i.e. $\left|q^{2}\right| \ll M_{\mathrm{H}}^{2}$. We mention that - owing to the gauge invariance of the Higgs field and its vacuum expectation value $v$-all parameter counterterms in the on-shell scheme are gauge-independent, i.e. independent of $\xi$, which is in contrast to the situation of a linearly realized Higgs sector [11]. Since $\delta M_{\mathrm{W}}^{2}, \delta t, \delta g$ are calculated from vertex functions at low-energy scales, their contributions arising from virtual Higgs exchange can be directly read from the effective Lagrangian (5.8), yielding

$$
\begin{aligned}
\delta M_{\mathrm{W}}^{2} & =\frac{1}{16 \pi^{2}} g^{2}\left(\frac{1}{4} I_{010}-I_{111}(1)\right)+\mathcal{O}\left(M_{\mathrm{H}}^{0}\right), \\
\delta t & =-\frac{1}{16 \pi^{2}} g \frac{3 M_{\mathrm{H}}^{2}}{4 M_{\mathrm{W}}} I_{010}+\mathcal{O}\left(M_{\mathrm{H}}^{0}\right), \\
\delta g & =\mathcal{O}\left(M_{\mathrm{H}}^{0}\right) .
\end{aligned}
$$

By definition the physical Higgs mass $M_{\mathrm{H}}$ is fixed at $q^{2}=M_{\mathrm{H}}^{2} \gg M_{\mathrm{W}}^{2}$ so that the counterterm $\delta M_{\mathrm{H}}^{2}$ has to be calculated diagrammatically as usual and cannot be read from (5.8). This is due to the fact that we apply on-shell renormalization. If one used a renormalization scheme in that $\delta M_{\mathrm{H}}^{2}$ is fixed at $\left|q^{2}\right| \ll M_{\mathrm{H}}^{2}$, also this renormalization constant could be calculated by functional methods. However, in such a renormalization scheme, $M_{\mathrm{H}}^{2}$ is not the physical Higgs mass. Thus, in order to construct the heavy-Higgs limit of the GNLSM, we find it most consistent to apply on-shell renormalization, where $M_{\mathrm{H}}^{2}$ is the physical Higgs mass, although this means that a very small part of our calculation 


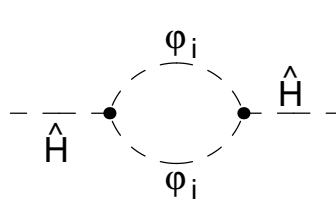

a)

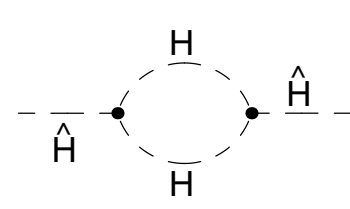

b)

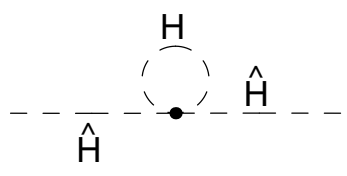

c)

Figure 1: All diagrams of order $\mathcal{O}\left(M_{\mathrm{H}}^{4}\right)$ in $\delta M_{\mathrm{H}}^{2}$.

has to be done diagrammatically. Since each background Higgs field contributes two inverse powers of $M_{\mathrm{H}}$ (see (4.11)), Re $\left\{\Sigma^{\hat{H}} \hat{H}\left(M_{\mathrm{H}}^{2}\right)\right\}$ in (6.4) has only to be evaluated in order $\mathcal{O}\left(M_{\mathrm{H}}^{4}\right)$. Hence, we just have to consider the diagrams shown in Fig. 1. We obtain

$$
\begin{aligned}
\delta M_{\mathrm{H}}^{2}= & \frac{1}{16 \pi^{2}} g^{2} \frac{3 M_{\mathrm{H}}^{2}}{8 M_{\mathrm{W}}^{2}}\left[M_{\mathrm{H}}^{2} \operatorname{Re}\left\{B_{0}\left(M_{\mathrm{H}}^{2}, 0,0\right)\right\}+3 M_{\mathrm{H}}^{2} B_{0}\left(M_{\mathrm{H}}^{2}, M_{\mathrm{H}}, M_{\mathrm{H}}\right)+I_{010}\right] \\
& +\mathcal{O}\left(M_{\mathrm{H}}^{2}\right),
\end{aligned}
$$

where $B_{0}$ denotes the general scalar two-point function,

$$
B_{0}\left(k^{2}, M_{0}, M_{1}\right)=\frac{(2 \pi \mu)^{4-D}}{i \pi^{2}} \int d^{D} p \frac{1}{\left[p^{2}-M_{0}^{2}+i \varepsilon\right]\left[(p+k)^{2}-M_{1}^{2}+i \varepsilon\right]} .
$$

The explicit expressions for the $B_{0}$-terms occurring in (6.7) are given in App. A. It should be noted, that in the non-linear parametrization of the GLSM, the $\hat{H} \varphi \varphi$-vertex is different from that in the linear one. Nevertheless, the contribution of the Goldstone loop to $\delta M_{\mathrm{H}}^{2}$ in this order is the same in both cases.

Finally, we introduce the field renormalization

$$
\begin{aligned}
\hat{W}^{ \pm} \rightarrow \hat{W}_{0}^{ \pm} & =Z_{\hat{W}}^{1 / 2} \hat{W}^{ \pm}=\left(1+\frac{1}{2} \delta Z_{\hat{W}}\right) \hat{W}^{ \pm}, \\
\hat{\varphi}^{ \pm} & \rightarrow \hat{\varphi}_{0}^{ \pm}=Z_{\hat{\varphi}}^{1 / 2} \hat{\varphi}^{ \pm}=\left(1+\frac{1}{2} \delta Z_{\hat{\varphi}}\right) \hat{\varphi}^{ \pm}, \\
\hat{H}^{ \pm} & \rightarrow \hat{H}_{0}=Z_{\hat{H}}^{1 / 2} \hat{H}=\left(1+\frac{1}{2} \delta Z_{\hat{H}}\right) \hat{H},
\end{aligned}
$$

for the background fields. In Ref. [11] it was demonstrated for the standard model that field renormalization constants can be chosen such that in the BFM the renormalized vertex functions obey the same Ward identities as the unrenormalized ones. Although this will be not of great importance in view of the heavy-Higgs limit, we note that this fact also holds in the non-linearly parametrized theory. Inspecting (2.2) and (2.6), one immediately concludes that the renormalized Lagrangian remains gauge-invariant if

$$
\begin{aligned}
\delta Z_{\hat{W}} & =-2 \frac{\delta g}{g}, \\
\delta Z_{\hat{\varphi}} & =2 \frac{\delta v}{v},
\end{aligned}
$$

which is equivalent to the requirement that the renormalized and unrenormalized vertex functions obey the same Ward identities. Since in the non-linear parametrization the Higgs 
field $\hat{H}$ is an $\mathrm{SU}(2)$ singlet, the $\hat{H}$ field-renormalization constant is not determined by this condition. On the other hand, $Z_{\hat{H}}$ will drop out anyhow if $\hat{H}$ is removed so that we can simply choose

$$
Z_{\hat{H}}=1, \quad \delta Z_{\hat{H}}=0 .
$$

In this context, we mention that the off-shell self-energy $\Sigma^{\hat{H}} \hat{H}\left(k^{2}\right)$ of $\hat{H}$ will even be UVdivergent for any value of $\delta Z_{\hat{H}}$ owing to the presence of UV-divergent terms proportional to $k^{4}$. Of course, the occurrence of such a term is an artefact of the non-linear parametrization of the Higgs sector since the complete theory is nevertheless renormalizable.

Applying finally the complete renormalization transformation (6.1), (6.9) to the Lagrangian (2.7), we obtain the $\hat{H}$-dependent part $\mathcal{L}_{\hat{\mathrm{H}}}^{\text {ct }}$ of the counterterm Lagrangian,

$$
\mathcal{L}_{\hat{\mathrm{H}}}^{\mathrm{ct}}=\delta t \hat{H}-\frac{1}{2} \delta M_{\mathrm{H}}^{2} \hat{H}^{2}-\frac{1}{2} \frac{\delta M_{\mathrm{W}}^{2}}{g M_{\mathrm{W}}} \hat{H} \operatorname{tr}\left\{\hat{V}^{\mu} \hat{V}_{\mu}\right\}+\mathcal{O}\left(\zeta^{-2}\right) .
$$

In slight abuse of terminology we call the sum of the effective Lagrangian $\mathcal{L}_{\text {eff }}$ and $\mathcal{L}_{\hat{\mathrm{H}}}^{\text {ct }}$ the renormalized effective Lagrangian $\mathcal{L}_{\text {eff }}^{\text {ren }}$. With the explicit expressions of (5.8), (6.6), (6.7), (6.10), and (6.11) we get the result

$$
\begin{aligned}
\mathcal{L}_{\text {eff }}^{\text {ren }}= & \frac{1}{16 \pi^{2}} \frac{3 g^{2} M_{\mathrm{H}}^{4}}{16 M_{\mathrm{W}}^{2}}\left(3 I_{020}-3 B_{0}\left(M_{\mathrm{H}}^{2}, M_{\mathrm{H}}, M_{\mathrm{H}}\right)-\operatorname{Re}\left\{B_{0}\left(M_{\mathrm{H}}^{2}, 0,0\right)\right\}\right) \hat{H}^{2} \\
& +\frac{1}{16 \pi^{2}} \frac{g}{8 M_{\mathrm{W}}}\left(-I_{010}+4 I_{111}(1)+3 M_{\mathrm{H}}^{2} I_{020}-12 M_{\mathrm{H}}^{2} I_{121}(\xi)\right) \hat{H} \operatorname{tr}\left\{\hat{V}_{\mu} \hat{V}^{\mu}\right\} \\
& +\frac{1}{16 \pi^{2}}\left(\frac{1}{4} I_{010}+M_{\mathrm{W}}^{2} I_{011}(1)-I_{111}(1)\right) \operatorname{tr}\left\{\hat{V}_{\mu} \hat{V}^{\mu}\right\} \\
& +\frac{1}{16 \pi^{2}} g^{2}\left(-\frac{1}{2} I_{112}(\xi)+2 I_{213}(\xi)\right) \operatorname{tr}\left\{\hat{W}_{\mu \nu} \hat{W}^{\mu \nu}\right\} \\
& +\frac{1}{16 \pi^{2}}\left(-I_{011}(\xi)+5 I_{112}(\xi)-12 I_{213}(\xi)\right) \operatorname{tr}\left\{\left(\hat{D}_{W}^{\mu} \hat{V}_{\mu}\right)^{2}\right\} \\
& +\frac{1}{16 \pi^{2}} i g\left(\frac{1}{2} I_{112}(\xi)-4 I_{213}(\xi)\right) \operatorname{tr}\left\{\hat{W}_{\mu \nu}\left[\hat{V}^{\mu}, \hat{V}^{\nu}\right]\right\} \\
& +\frac{1}{16 \pi^{2}}\left(-4 I_{213}(\xi)+2 I_{222}(\xi)\right) \operatorname{tr}\left\{\hat{V}_{\mu} \hat{V}_{\nu}\right\} \operatorname{tr}\left\{\hat{V}^{\mu} \hat{V}^{\nu}\right\} \\
& +\frac{1}{16 \pi^{2}}\left(\frac{1}{16} I_{020}-\frac{1}{2} I_{121}(\xi)+4 I_{213}(\xi)+I_{222}(\xi)\right)\left(\operatorname{tr}\left\{\hat{V}_{\mu} \hat{V}^{\mu}\right\}\right)^{2} \\
& +\mathcal{O}\left(\zeta^{-2}\right),
\end{aligned}
$$

where the (tadpole) terms linear in $\hat{H}$ drop out as expected.

\section{Elimination of the background Higgs field}

After having integrated out the quantum Higgs field $H$, the effective Lagrangian (6.13) still contains the background Higgs field $\hat{H}$. Integrating out the quantum Higgs corresponds in the diagrammatical formalism to the calculation of the effects of the heavy Higgs boson in loops. The elimination of the background Higgs field yields the effects of the Higgs field outside loops. 
The background fields occur as tree lines in the diagrammatical calculation of (reducible) Green functions and S-matrix elements. For $M_{\mathrm{H}} \rightarrow \infty$ no external $\hat{H}$ fields occur, and (internal) $\hat{H}$ propagators can be expanded in powers of $1 / M_{\mathrm{H}}^{2}$. Diagrammatically this means that the $H$ propagator shrinks to a point rendering such (sub-)graphs irreducible which contain $\hat{H}$ lines only. Inspecting the $\hat{H}$-terms in the Lagrangian of the GLSM, one easily finds that this expansion leads to the replacement

$$
\hat{H} \rightarrow-\frac{M_{\mathrm{W}}}{g M_{\mathrm{H}}^{2}} \operatorname{tr}\left\{\hat{V}_{\mu} \hat{V}^{\mu}\right\}+\mathcal{O}\left(M_{\mathrm{H}}^{-4}\right) .
$$

This substitution can also be motivated as follows. The tree-like parts of Feynman graphs correspond to the lowest order in the perturbative expansion of amplitudes which is known to agree with the lowest-order result of the classical equations of motion (EOM). The EOM for the $\hat{H}$ field is given by

$$
\left(\partial^{2}+M_{\mathrm{H}}^{2}\right) \hat{H}=-\frac{M_{\mathrm{W}}}{g} \operatorname{tr}\left\{\hat{V}_{\mu} \hat{V}^{\mu}\right\}-\frac{1}{2} \hat{H} \operatorname{tr}\left\{\hat{V}_{\mu} \hat{V}^{\mu}\right\}-\frac{3 g M_{\mathrm{H}}^{2}}{4 M_{\mathrm{W}}} \hat{H}^{2}-\frac{g^{2} M_{\mathrm{H}}^{2}}{8 M_{\mathrm{W}}^{2}} \hat{H}^{3},
$$

which can be solved for $\hat{H}$ by recursion. The leading contribution exactly corresponds to (7.1).

First, we insert (7.1) into the tree-level Lagrangian of the GLSM, i.e. that part of (2.7) that contains only background fields:

$$
\begin{aligned}
\mathcal{L}^{\text {tree }}= & -\frac{1}{2} \operatorname{tr}\left\{\hat{W}_{\mu \nu} \hat{W}^{\mu \nu}\right\}+\frac{1}{2}\left(\partial_{\mu} \hat{H}\right)\left(\partial^{\mu} \hat{H}\right)-\frac{1}{4}\left(\frac{2 M_{\mathrm{W}}}{g}+\hat{H}\right)^{2} \operatorname{tr}\left\{\hat{V}_{\mu} \hat{V}^{\mu}\right\} \\
& -\frac{1}{2} M_{\mathrm{H}}^{2} \hat{H}^{2}-\frac{g^{2} M_{\mathrm{H}}^{2}}{4 M_{\mathrm{W}}} \hat{H}^{3}-\frac{g^{2} M_{\mathrm{H}}^{2}}{32 M_{\mathrm{W}}^{2}} \hat{H}^{4} .
\end{aligned}
$$

One immediately finds that in $\mathcal{O}\left(M_{\mathrm{H}}^{0}\right)$ (7.1) results in simply dropping $\hat{H}$ in (7.3). This is the well-known result that the limit for $M_{\mathrm{H}} \rightarrow \infty$ of the gauged linear $\sigma$-model at tree level is the gauged non-linear $\sigma$-model [14]:

$$
\left.\mathcal{L}^{\text {tree }}\right|_{M_{\mathrm{H}} \rightarrow \infty}=\left.\mathcal{L}^{\text {tree }}\right|_{\hat{H}=0}+\mathcal{O}\left(M_{\mathrm{H}}^{-2}\right)=\mathcal{L}_{\mathrm{GNLSM}}^{\text {tree }}+\mathcal{O}\left(M_{\mathrm{H}}^{-2}\right),
$$

with

$$
\mathcal{L}_{\mathrm{GNLSM}}^{\mathrm{tree}}=-\frac{1}{2} \operatorname{tr}\left\{\hat{W}_{\mu \nu} \hat{W}^{\mu \nu}\right\}-\frac{M_{\mathrm{W}}^{2}}{g^{2}} \operatorname{tr}\left\{\hat{V}_{\mu} \hat{V}^{\mu}\right\} .
$$

Eq. (4.1) implies that the one-loop Lagrangian of the GLSM for $M_{\mathrm{H}} \rightarrow \infty$ consists of two parts, namely the effective Lagrangian $\mathcal{L}_{\text {eff }}^{\text {ren }}$ of $(6.13)$ and the remainder $\left.\mathcal{L}^{1-\text { loop }}\right|_{H=0}$ in the path integral (4.1). The effective Lagrangian was generated by integrating out the quantum Higgs, i.e. it parametrizes the effects of loops containing the heavy Higgs bosons. The remainder Lagrangian $\left.\mathcal{L}^{1-\text { loop }}\right|_{H=0}$ still contains the light quantum fields, i.e. it has to be used in order to calculate the contributions from loops without the heavy Higgs boson. As in the case of the tree-level Lagrangian, in $\mathcal{O}\left(M_{\mathrm{H}}^{0}\right)$ the application of substitution (7.1) simply results in dropping $\hat{H}$ in $\left.\mathcal{L}^{1-\text { loop }}\right|_{H=0}$, which yields the one-loop Lagrangian of GNLSM. The effective Lagrangian (6.13), however, parametrizes the differences of the 
GLSM for $M_{\mathrm{H}} \rightarrow \infty$ and the GNLSM. Applying (7.1) to (6.13) yields non-vanishing contributions at $\mathcal{O}\left(M_{\mathrm{H}}^{0}\right)$. One finds

$$
\begin{aligned}
\mathcal{L}_{\text {eff }}^{\text {ren }}= & \frac{1}{16 \pi^{2}}\left(\frac{1}{4} I_{010}+M_{\mathrm{W}}^{2} I_{011}(1)-I_{111}(1)\right) \operatorname{tr}\left\{\hat{V}_{\mu} \hat{V}^{\mu}\right\} \\
& +\frac{1}{16 \pi^{2}}\left(-\frac{1}{2} I_{112}(\xi)+2 I_{213}(\xi)\right) g^{2} \operatorname{tr}\left\{\hat{W}_{\mu \nu} \hat{W}^{\mu \nu}\right\} \\
& +\frac{1}{16 \pi^{2}}\left(-I_{011}(\xi)+5 I_{112}(\xi)-12 I_{213}(\xi)\right) \operatorname{tr}\left\{\left(\hat{D}_{W}^{\mu} \hat{V}_{\mu}\right)^{2}\right\} \\
& +\frac{1}{16 \pi^{2}}\left(\frac{1}{2} I_{112}(\xi)-4 I_{213}(\xi)\right) \operatorname{ig} \operatorname{tr}\left\{\hat{W}_{\mu \nu}\left[\hat{V}^{\mu}, \hat{V}^{\nu}\right]\right\} \\
& +\frac{1}{16 \pi^{2}}\left(-4 I_{213}(\xi)+2 I_{222}(\xi)\right) \operatorname{tr}\left\{\hat{V}_{\mu} \hat{V}_{\nu}\right\} \operatorname{tr}\left\{\hat{V}^{\mu} \hat{V}^{\nu}\right\} \\
& +\frac{1}{16 \pi^{2}}\left(\frac{1}{8 M_{\mathrm{H}}^{2}} I_{010}-\frac{1}{2 M_{\mathrm{H}}^{2}} I_{111}(1)+\frac{1}{4} I_{020}+I_{121}(\xi)+4 I_{213}(\xi)+I_{222}(\xi)\right. \\
& \left.\quad-\frac{9}{16} B_{0}\left(M_{\mathrm{H}}^{2}, M_{\mathrm{H}}, M_{\mathrm{H}}\right)-\frac{3}{16} \operatorname{Re}\left\{B_{0}\left(M_{\mathrm{H}}^{2}, 0,0\right)\right\}\right)\left(\operatorname{tr}\left\{\hat{V}_{\mu} \hat{V}^{\mu}\right\}\right)^{2} \\
& +\mathcal{O}\left(M_{\mathrm{H}}^{-2}\right) .
\end{aligned}
$$

Inserting the explicit expressions (A.2) and (A.4) for the integrals occurring in (7.6) we finally find for $\mathcal{L}_{\text {eff }}^{\text {ren }}$ :

$$
\begin{aligned}
\mathcal{L}_{\text {eff }}^{\text {ren }}= & \frac{1}{16 \pi^{2}}\left[-\frac{1}{8} M_{\mathrm{H}}^{2}+\frac{3}{4} M_{\mathrm{W}}^{2}\left(\Delta_{M_{\mathrm{H}}}+\frac{5}{6}\right)\right] \operatorname{tr}\left\{\hat{V}_{\mu} \hat{V}^{\mu}\right\} \\
& -\frac{1}{16 \pi^{2}} \frac{1}{24}\left(\Delta_{M_{\mathrm{H}}}+\frac{5}{6}\right) g^{2} \operatorname{tr}\left\{\hat{W}_{\mu \nu} \hat{W}^{\mu \nu}\right\} \\
& -\frac{1}{16 \pi^{2}} \frac{1}{4}\left(\Delta_{M_{\mathrm{H}}}+\frac{1}{6}\right) \operatorname{tr}\left\{\left(\hat{D}_{W}^{\mu} \hat{V}_{\mu}\right)^{2}\right\} \\
& -\frac{1}{16 \pi^{2}} \frac{1}{24}\left(\Delta_{M_{\mathrm{H}}}+\frac{17}{6}\right) i g \operatorname{tr}\left\{\hat{W}_{\mu \nu}\left[\hat{V}^{\mu}, \hat{V}^{\nu}\right]\right\} \\
& -\frac{1}{16 \pi^{2}} \frac{1}{12}\left(\Delta_{M_{\mathrm{H}}}+\frac{17}{6}\right) \operatorname{tr}\left\{\hat{V}_{\mu} \hat{V}_{\nu}\right\} \operatorname{tr}\left\{\hat{V}^{\mu} \hat{V}^{\nu}\right\} \\
& -\frac{1}{16 \pi^{2}} \frac{1}{24}\left(\Delta_{M_{\mathrm{H}}}+\frac{79}{3}-\frac{27 \pi}{2 \sqrt{3}}\right)\left(\operatorname{tr}\left\{\hat{V}_{\mu} \hat{V}^{\mu}\right\}\right)^{2} \\
& +\mathcal{O}\left(M_{\mathrm{H}}^{-2}\right),
\end{aligned}
$$

with $\Delta_{M_{\mathrm{H}}}$ being given in (A.3).

As a final result for the one-loop Lagrangian of the GLSM for $M_{\mathrm{H}} \rightarrow \infty$ we find:

$$
\begin{aligned}
\left.\mathcal{L}^{1-\text { loop,ren }}\right|_{M_{\mathrm{H}} \rightarrow \infty} & =\left.\mathcal{L}^{1-\text { loop }}\right|_{H=\hat{H}=0}+\left.\mathcal{L}^{\mathrm{ct}}\right|_{\hat{H}=0}+\mathcal{L}_{\text {eff }}^{\text {ren }}+\mathcal{O}\left(M_{\mathrm{H}}^{-2}\right) \\
& =\mathcal{L}_{\mathrm{GNLSM}}^{1-\text { loop }}+\left.\mathcal{L}^{\mathrm{ct}}\right|_{\hat{H}=0}+\mathcal{L}_{\text {eff }}^{\text {ren }}+\mathcal{O}\left(M_{\mathrm{H}}^{-2}\right) .
\end{aligned}
$$

In (7.8) the counterterm Lagrangian $\left.\mathcal{L}^{\mathrm{ct}}\right|_{\hat{H}=0}$ simply follows from the tree-level GNLSM Lagrangian (77.5) upon applying the renormalization transformations for $v, M_{\mathrm{W}}, g, \hat{\varphi}$, and $\hat{W}$ (see (6.1), (6.2), (6.3), and (6.9)), where $\delta M_{\mathrm{W}}^{2}$ is fixed by the on-shell condition (6.4), 
and $\delta Z_{\hat{\varphi}}$ by (6.10). We did not make use of a specification for $\delta g$ and $\delta Z_{\hat{W}}$ so that these counterterms can be chosen independently. Note that the contributions of the effective Lagrangian $\mathcal{L}_{\text {eff }}^{\text {ren }}(7.8)$ have to be included in the determination of the counterterms.

The effective Lagrangian $\mathcal{L}_{\text {eff }}^{\text {ren }}$ of $(7.7)$ quantifies the exact difference between the heavyHiggs GLSM (in non-linear representation of the Higgs sector) and the GNLSM at one loop. More precisely, applying (7.7) one obtains the difference for each vertex function, where the tadpole and Higgs-mass renormalization has already been carried out in the GLSM, but the remaining renormalization is still to be done. Inspecting (7.7), one finds that the first two terms have the same structure as terms in the tree-level Lagrangian of the GNLSM (7.5). Thus, the contributions of the $\operatorname{tr}\left\{\hat{V}_{\mu} \hat{V}^{\mu}\right\}$ and $\operatorname{tr}\left\{\hat{W}_{\mu \nu} \hat{W}^{\mu \nu}\right\}$-terms in (7.7) can be absorbed into the corresponding counterterms. This means that S-matrix elements are not influenced by these terms.

Furthermore, it should be noted that the $\operatorname{tr}\left\{\left(\hat{D}_{W}^{\mu} \hat{V}_{\mu}\right)^{2}\right\}$-term in (7.7) is redundant in view of the calculation of (reducible) Green functions and S-matrix elements. Actually, we may not only use the EOM for $\hat{H}$ but also those for $\hat{W}^{\mu}$ in order to simplify the effective interaction term $\mathcal{L}_{\text {eff }}^{\text {ren }}$, although in the latter case this does not correspond to a $1 / M_{\mathrm{H}^{-}}$expansion. The reason for this is that the application of the lowest-order EOM in $\mathcal{L}_{\text {eff }}^{\text {ren }}$ simply corresponds to a field transformation of the background fields, which leaves S-matrix elements invariant, and to an expansion in the coupling constant of the effective interaction term (i.e. in $g^{2} /\left(16 \pi^{2}\right)$ in our case) [19]. However, the EOM for the vector fields in the GNLSM

$$
\hat{D}_{W}^{\mu} \hat{W}_{\mu \nu}=-\frac{i}{g} M_{\mathrm{W}}^{2} \hat{V}_{\nu}
$$

implies

$$
\hat{D}_{W}^{\mu} \hat{V}_{\mu}=0
$$

Hence, the contributions of the $\operatorname{tr}\left\{\left(\hat{D}_{W}^{\mu} \hat{V}_{\mu}\right)^{2}\right\}$-term drop out in complete (reducible) Green functions and S-matrix elements, even though this term yields non-vanishing contributions to single vertex functions. Consequently, the effective Lagrangian

$$
\begin{aligned}
\mathcal{L}_{\text {eff }}^{\text {ren }}(\text { S-matrix })= & -\frac{1}{16 \pi^{2}} \frac{1}{24}\left(\Delta_{M_{\mathrm{H}}}+\frac{17}{6}\right) i g \operatorname{tr}\left\{\hat{W}_{\mu \nu}\left[\hat{V}^{\mu}, \hat{V}^{\nu}\right]\right\} \\
& -\frac{1}{16 \pi^{2}} \frac{1}{12}\left(\Delta_{M_{\mathrm{H}}}+\frac{17}{6}\right) \operatorname{tr}\left\{\hat{V}_{\mu} \hat{V}_{\nu}\right\} \operatorname{tr}\left\{\hat{V}^{\mu} \hat{V}^{\nu}\right\} \\
& -\frac{1}{16 \pi^{2}} \frac{1}{24}\left(\Delta_{M_{\mathrm{H}}}+\frac{79}{3}-\frac{27 \pi}{2 \sqrt{3}}\right)\left(\operatorname{tr}\left\{\hat{V}_{\mu} \hat{V}^{\mu}\right\}\right)^{2} \\
& +\mathcal{O}\left(M_{\mathrm{H}}^{-2}\right)
\end{aligned}
$$

summarizes the complete differences between the GLSM and the GNLSM contributing to the S-matrix.

\section{Some examples of vertex functions}

In order to illustrate our results, we consider some special vertex functions and calculate their difference between the GLSM with a heavy Higgs boson and the corresponding 
GNLSM. For these examples we compare the results derived from the effective Lagrangian (7.6) with the ones obtained by evaluating directly the Feynman diagrams. Note that if the non-linear parametrization, (2.5) with (2.6), is used for the GLSM, the difference between the GLSM and the GNLSM in diagrammatical calculations comes only from diagrams with internal Higgs lines. The situation will be different if the linear parametrization (2.3) is used, as in Ref. [1], where also some diagrams without Higgs lines differ in both models.

Instead of using the fields $W_{i}$ and $\varphi_{i}$ as introduced in Sect. 2, we find it convenient to introduce the fields

$$
\begin{aligned}
W_{\mu}^{ \pm} & =\frac{1}{\sqrt{2}}\left(W_{\mu}^{1} \mp i W_{\mu}^{2}\right), & W_{\mu}^{0} & =W_{\mu}^{3}, \\
\varphi^{ \pm} & =\frac{1}{\sqrt{2}}\left(\varphi_{2} \pm i \varphi_{1}\right), & \chi & =-\varphi_{3} .
\end{aligned}
$$

We mention that the definitions of $W_{\mu}^{ \pm}, \varphi^{ \pm}$, and $\chi$ follow the ones for the SM fields of Refs. 10, 11, 18, where the linear parametrization (2.3) is used. The SM $Z$-field introduced there reduces to our $W^{0}$-field upon replacing the couplings $g_{2} \rightarrow g, g_{1} \rightarrow 0$.

We start by considering the $\hat{W}^{0}$ self-energy. In Fig. 2 we show the corresponding Feynman graphs which contain the Higgs field in the GLSM, but are absent in the GNLSM. As mentioned above, the graphs of Fig. 2 form exactly the difference between the GLSM (with non-linear Higgs realization) and the GNLSM. We mention that we have not explicitly written down the tadpole graph and its counterterm, since these terms obviously cancel. Calculation of each graph yields

$$
\begin{aligned}
& \left.\Gamma_{\mu \nu}^{\hat{W}^{0} \hat{W}^{0}}\left(k_{0}\right)\right|_{2 \mathrm{a})}=\mu^{4-D} \int \frac{d^{D} p}{(2 \pi)^{D}} \frac{\left(-g^{2}\right) M_{\mathrm{W}}^{2}}{p^{2}-M_{\mathrm{H}}^{2}} \\
& \times\left\{\frac{g_{\mu \nu}}{\left(p+k_{0}\right)^{2}-M_{\mathrm{W}}^{2}}-\frac{(1-\xi)\left(p+k_{0}\right)_{\mu}\left(p+k_{0}\right)_{\nu}}{\left[\left(p+k_{0}\right)^{2}-M_{\mathrm{W}}^{2}\right]\left[\left(p+k_{0}\right)^{2}-\xi M_{\mathrm{W}}^{2}\right]}\right\} \\
& =-\frac{i g^{2}}{16 \pi^{2}} g_{\mu \nu}\left\{M_{\mathrm{W}}^{2} I_{011}(1)+I_{111}(\xi)-I_{111}(1)\right\}+\mathcal{O}\left(M_{\mathrm{H}}^{-2}\right), \\
& \left.\Gamma_{\mu \nu}^{\hat{W}^{0} \hat{W}^{0}}\left(k_{0}\right)\right|_{\text {国 })}=\mu^{4-D} \int \frac{d^{D} p}{(2 \pi)^{D}} \frac{g^{2}\left(p+k_{0}\right)_{\mu}\left(p+k_{0}\right)_{\nu}}{\left[p^{2}-M_{\mathrm{H}}^{2}\right]\left[\left(p+k_{0}\right)^{2}-\xi M_{\mathrm{W}}^{2}\right]} \\
& =\frac{i g^{2}}{16 \pi^{2}}\left\{g_{\mu \nu}\left[I_{111}(\xi)-k_{0}^{2} I_{112}(\xi)+4 k_{0}^{2} I_{213}(\xi)\right]\right. \\
& \left.+k_{0, \mu} k_{0, \nu}\left[I_{011}(\xi)-4 I_{112}(\xi)+8 I_{213}(\xi)\right]\right\}+\mathcal{O}\left(M_{\mathrm{H}}^{-2}\right), \\
& \left.\Gamma_{\mu \nu}^{\hat{W}^{0} \hat{W}^{0}}\left(k_{0}\right)\right|_{\text {[ec })}=\mu^{4-D} \int \frac{d^{D} p}{(2 \pi)^{D}} \frac{\left(-g^{2}\right) g_{\mu \nu}}{4\left(p^{2}-M_{\mathrm{H}}^{2}\right)} \\
& =-\frac{i g^{2}}{16 \pi^{2}} \frac{1}{4} g_{\mu \nu} I_{010}
\end{aligned}
$$




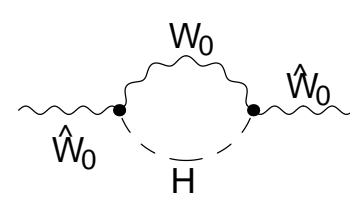

a)

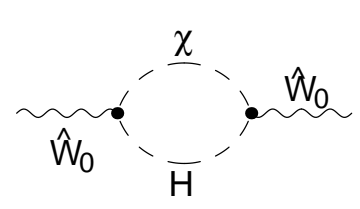

b)

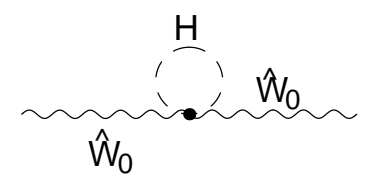

C)

Figure 2: Higgs diagrams for the $\hat{W}^{0}$ self-energy.

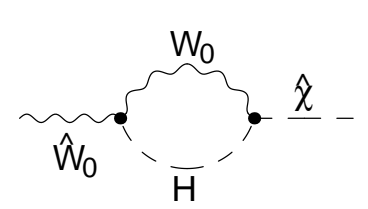

a)

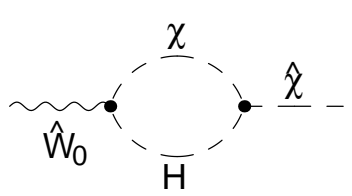

b)

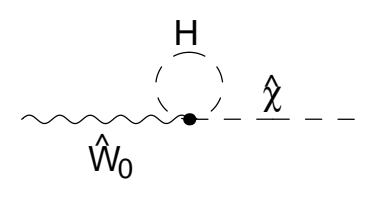

C)

Figure 3: Higgs diagrams for the $\hat{W}^{0} \hat{\chi}$-mixing self-energy.

where we have already performed the $1 / M_{\mathrm{H}}$-expansions by expanding the Feynman integrals around the vacuum integrals defined in (4.26). Adding up terms 2a)-2ac), one obtains

$$
\begin{gathered}
\delta \Gamma_{\mu \nu}^{\hat{W}^{0} \hat{W}^{0}}\left(k_{0}\right)=\frac{i g^{2}}{16 \pi^{2}}\left\{g_{\mu \nu}\left[I_{111}(1)-M_{\mathrm{W}}^{2} I_{011}(1)-\frac{1}{4} I_{010}-k_{0}^{2} I_{112}(\xi)+4 k_{0}^{2} I_{213}(\xi)\right]\right. \\
\left.+k_{0, \mu} k_{0, \nu}\left[I_{011}(\xi)-4 I_{112}(\xi)+8 I_{213}(\xi)\right]\right\}+\mathcal{O}\left(M_{\mathrm{H}}^{-2}\right) .
\end{gathered}
$$

This result has to be compared with the contribution of the effective Lagrangian (7.6) to the $\hat{W}^{0}$ self-energy. To this end, we give the $\hat{W}^{0} \hat{W}^{0}$-parts of the relevant traces occurring in (7.6) so that one can easily read off the Feynman rules,

$$
\begin{aligned}
& \left.\operatorname{tr}\left\{\hat{W}_{\mu \nu} \hat{W}^{\mu \nu}\right\}\right|_{\hat{W}^{0} \hat{W}^{0}}=-\left(\partial \hat{W}^{0}\right)^{2}-\hat{W}_{\mu}^{0} \partial^{2} \hat{W}^{0, \mu} \\
& \left.\operatorname{tr}\left\{\left(\hat{D}_{W}^{\mu} \hat{V}_{\mu}\right)^{2}\right\}\right|_{\hat{W}^{0} \hat{W}^{0}}=-\frac{g^{2}}{2}\left(\partial \hat{W}^{0}\right)^{2} \\
& \left.\quad \operatorname{tr}\left\{\hat{V}_{\mu} \hat{V}^{\mu}\right\}\right|_{\hat{W}^{0} \hat{W}^{0}}=-\frac{g^{2}}{2}\left(\hat{W}^{0}\right)^{2} .
\end{aligned}
$$

Using (7.6) with (8.4) one directly obtains (8.3).

Next, we turn to the $\hat{W}^{0} \hat{\chi}$-mixing self-energy. Fig. 3 shows the diagrams for the difference between the GLSM and the GNLSM in analogy to the previous example. The tadpoles (which cancel) are again not explicitly drawn. The individual graphs are calculated to

$$
\begin{aligned}
\left.\Gamma_{\mu}^{\hat{W}^{0} \hat{\chi}}\left(k_{0}\right)\right|_{3 \mathrm{a})}=\mu^{4-D} & \int \frac{d^{D} p}{(2 \pi)^{D}} \frac{\left(-i g^{2}\right) M_{\mathrm{W}} k_{0, \alpha}}{p^{2}-M_{\mathrm{H}}^{2}} \\
& \times\left\{\frac{g_{\mu \alpha}}{\left(p+k_{0}\right)^{2}-M_{\mathrm{W}}^{2}}-\frac{(1-\xi)\left(p+k_{0}\right)_{\mu}\left(p+k_{0}\right)_{\alpha}}{\left[\left(p+k_{0}\right)^{2}-M_{\mathrm{W}}^{2}\right]\left[\left(p+k_{0}\right)^{2}-\xi M_{\mathrm{W}}^{2}\right]}\right\}
\end{aligned}
$$




$$
\begin{aligned}
& =\frac{g^{2}}{16 \pi^{2}} \frac{k_{0, \mu}}{M_{\mathrm{W}}}\left\{M_{\mathrm{W}}^{2} I_{011}(1)+I_{111}(\xi)-I_{111}(1)\right\}+\mathcal{O}\left(M_{\mathrm{H}}^{-2}\right), \\
\Gamma_{\mu}^{\hat{W}^{0} \hat{\chi}}\left(k_{0}\right)_{\text {(3) })} & =\mu^{4-D} \int \frac{d^{D} p}{(2 \pi)^{D}} \frac{i g^{2}}{M_{\mathrm{W}}} \frac{\left(p+k_{0}\right)_{\mu}\left(p k_{0}+k_{0}^{2}\right)}{\left[p^{2}-M_{\mathrm{H}}^{2}\right]\left[\left(p+k_{0}\right)^{2}-\xi M_{\mathrm{W}}^{2}\right]} \\
& =-\frac{g^{2}}{16 \pi^{2}} \frac{k_{0, \mu}}{M_{\mathrm{W}}}\left\{I_{111}(\xi)+k_{0}^{2} I_{011}(1)-5 k_{0}^{2} I_{112}(\xi)+12 k_{0}^{2} I_{213}(\xi)\right\}+\mathcal{O}\left(M_{\mathrm{H}}^{-2}\right), \\
\Gamma_{\mu}^{\hat{W}^{0} \hat{\chi}}\left(k_{0}\right)_{\text {(3) })} & =\mu^{4-D} \int \frac{d^{D} p}{(2 \pi)^{D}} \frac{\left(-i g^{2}\right) k_{0, \mu}}{4 M_{\mathrm{W}}\left(p^{2}-M_{\mathrm{H}}^{2}\right)} \\
& =\frac{g^{2}}{16 \pi^{2}} \frac{k_{0, \mu}}{4 M_{\mathrm{W}}} I_{010} .
\end{aligned}
$$

The resulting contribution

$$
\begin{aligned}
\delta \Gamma_{\mu}^{\hat{W}^{0}} \hat{\chi}\left(k_{0}\right)=\frac{g^{2}}{16 \pi^{2}} \frac{k_{0, \mu}}{M_{\mathrm{W}}}\{ & M_{\mathrm{W}}^{2} I_{011}(1)-I_{111}(1)+\frac{1}{4} I_{010} \\
& \left.-k_{0}^{2} I_{011}(\xi)+5 k_{0}^{2} I_{112}(\xi)-12 k_{0}^{2} I_{213}(\xi)\right\}+\mathcal{O}\left(M_{\mathrm{H}}^{-2}\right)
\end{aligned}
$$

again agrees with the result from the effective Lagrangian (7.6). The necessary Feynman rules for the effective $\hat{W}^{0} \hat{\chi}$-mixing follow by inserting the corresponding terms

$$
\begin{gathered}
\left.\operatorname{tr}\left\{\hat{V}_{\mu} \hat{V}^{\mu}\right\}\right|_{\hat{W}^{0} \hat{\chi}}=-\frac{g^{2}}{M_{\mathrm{W}}}\left(\partial^{\mu} \hat{\chi}\right) \hat{W}_{\mu}^{0}, \\
\left.\operatorname{tr}\left\{\left(\hat{D}_{W}^{\mu} \hat{V}_{\mu}\right)^{2}\right\}\right|_{\hat{W}^{0} \hat{\chi}}=-\frac{g^{2}}{M_{\mathrm{W}}}\left(\partial^{2} \hat{\chi}\right)\left(\partial \hat{W}^{0}\right)
\end{gathered}
$$

into (7.6), and one immediately obtains (8.6). We remark that the differences $\delta \Gamma_{\mu \nu}^{\hat{W}^{0} \hat{W}^{0}}$ and $\delta \Gamma_{\mu}^{\hat{W}^{0}} \hat{\chi}$ obey the Ward identity

$$
0=k_{0}^{\mu} \Gamma_{\mu \nu}^{\hat{W}^{0} \hat{W}^{0}}\left(k_{0}\right)+i M_{\mathrm{W}} \Gamma_{\nu}^{\hat{W}^{0} \hat{\chi}}\left(k_{0}\right),
$$

which is fulfilled both in the GLSM with non-linearly realized scalar sector and the GNSLM.

Finally, we inspect the less trivial example of the four-point function $\Gamma_{\mu \nu \rho \sigma}^{\hat{W}^{+} \hat{W}^{-} \hat{W}^{0} \hat{W}^{0}}$. The heavy-Higgs contributions to $\Gamma_{\mu \nu \rho \sigma}^{\hat{W}^{+} \hat{W}^{-} \hat{W}^{0} \hat{W}^{0}}$ in the GLSM can be classified into three topologically different types: irreducible diagrams (Fig. (t), and reducible diagrams with either one (Fig. 5) or two (Fig. 6) $\hat{H}$ fields on tree lines. Again all tadpole terms cancel and are omitted from the beginning. In Figs. 46 we only show the diagrams which are at least of order $\mathcal{O}\left(M_{\mathrm{H}}^{0}\right)$; the (numerous) graphs of order $\mathcal{O}\left(M_{\mathrm{H}}^{-2}\right)$ are omitted.

The irreducible graphs are calculated to

$$
\begin{aligned}
\left.\delta \Gamma_{\mu \nu \rho \sigma}^{\hat{W}^{+} \hat{W}^{-} \hat{W}^{0} \hat{W}^{0}}\left(k_{+}, k_{-}, k_{0,1}, k_{0,2}\right)\right|_{\text {irr }}= & -\frac{i g^{4}}{16 \pi^{2}} g_{\mu \nu} g_{\rho \sigma}\left[I_{121}(\xi)-\frac{1}{8} I_{020}-2 I_{222}(\xi)\right] \\
& +\frac{i g^{4}}{16 \pi^{2}}\left(g_{\mu \rho} g_{\nu \sigma}+g_{\mu \sigma} g_{\nu \rho}\right) 2 I_{222}(\xi) .
\end{aligned}
$$

\footnotetext{
${ }^{3}$ Note that Feynman diagrams with tree lines of background fields other than $\hat{H}$ give no contributions to (irreducible!) vertex functions, since only the $\hat{H}$ propagator shrinks to a point for $M_{\mathrm{H}} \rightarrow \infty$.
} 


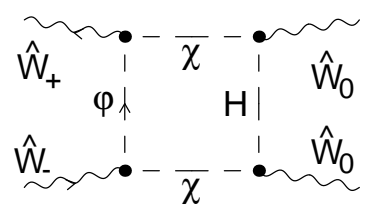

a)

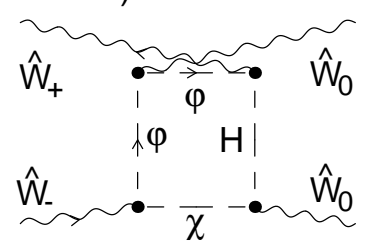

e)

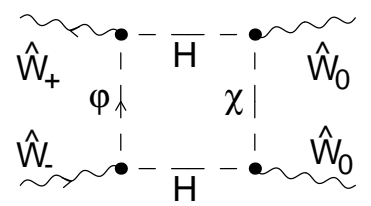

i)

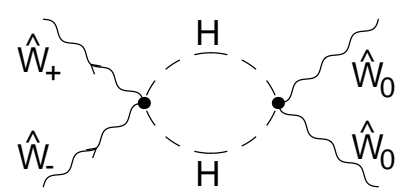

m)

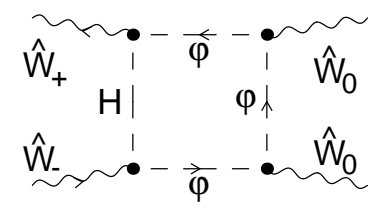

b)

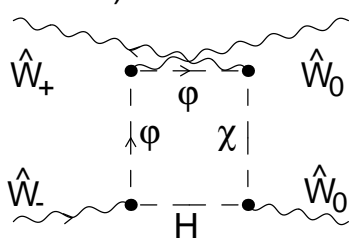

f)

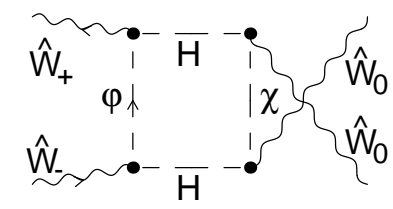

j)

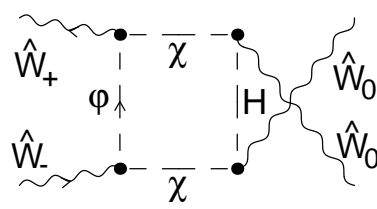

c)

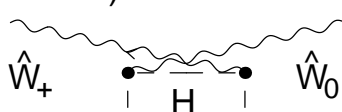

$\mid \chi \quad \varphi_{\text {小 }}$

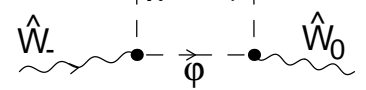

g)

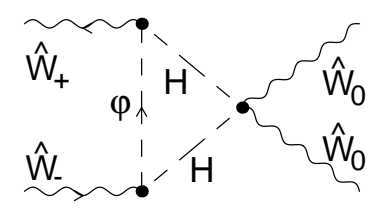

k)

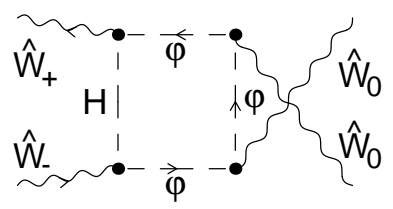

d)

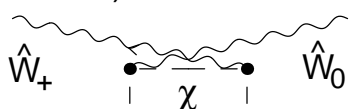

$\mid \mathrm{H} \quad \varphi_{\uparrow}$

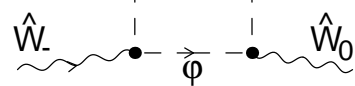

h)

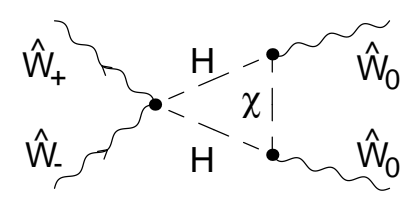

I)

Figure 4: Irreducible Higgs diagrams for the $\hat{W}^{+} \hat{W}^{-} \hat{W}^{0} \hat{W}^{0}$ four-point-function.

Instead of writing down the explicit expression for each diagram, we add some remarks on the single contributions. Inspecting the momentum integrals, one finds that the external momenta $k_{ \pm}, k_{0,1 / 2}$ do not give contributions of $\mathcal{O}\left(M_{\mathrm{H}}^{0}\right)$ and can be set to zero for all diagrams of Fig. 1 . Hence, all diagrams can be expressed in terms of $I_{k l m}(\xi)$-functions defined in (4.26). The $I_{213}(\xi)$-terms, which originate from graphs ta)-h), exactly cancel each other.

The sum of all diagrams involving exactly one background Higgs propagator (Fig. 5) is given by

$$
\begin{aligned}
&\left.\delta \Gamma_{\mu \nu \rho \sigma}^{\hat{W}^{+} \hat{W}^{-} \hat{W}^{0} \hat{W}^{0}}\left(k_{+}, k_{-}, k_{0,1}, k_{0,2}\right)\right|_{\hat{H}} \\
& \quad=\frac{i g^{4}}{16 \pi^{2}} g_{\mu \nu} g_{\rho \sigma}\left[3 I_{121}(\xi)-\frac{3}{4} I_{020}+\frac{1}{M_{\mathrm{H}}^{2}}\left(\frac{1}{4} I_{010}-I_{111}(\xi)\right)\right]
\end{aligned}
$$

The W-mass counterterm $\delta M_{\mathrm{W}}^{2}$ originates from the renormalization of the $\hat{H} \hat{W}^{+} \hat{W}^{-}$and $\hat{H} \hat{W}^{0} \hat{W}^{0}$ couplings, which is indicated by the graphs 5 $\left.5 \mathrm{k}\right), 1$ ), and is explicitly given in (6.6). Again, the external momenta do not contribute in $\mathcal{O}\left(M_{\mathrm{H}}^{0}\right)$. Note also that the diagrams 5(a),e),f) and 5(c),g),h), which yield contributions of $I_{111}(\xi)$, cancel each other, respectively. 


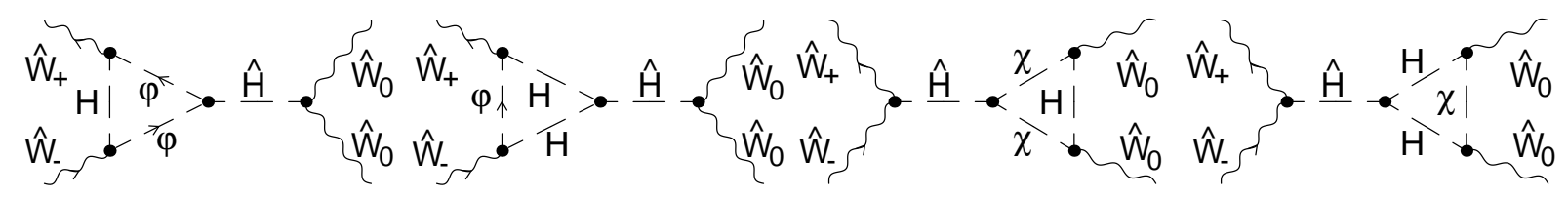

a)

b)

c)

d)

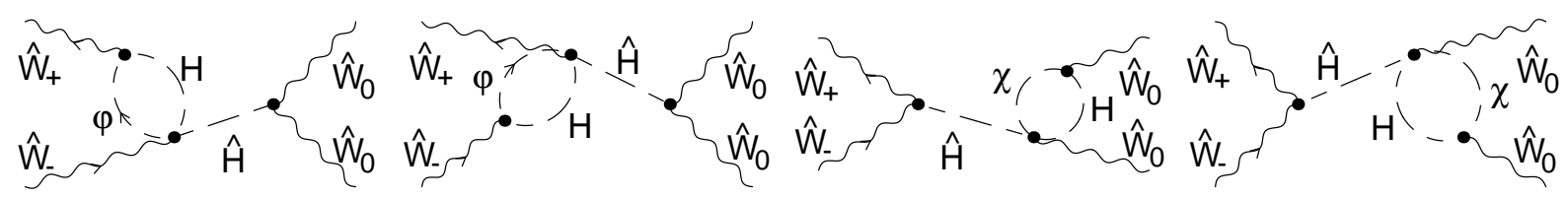

e)

f)

g)

h)

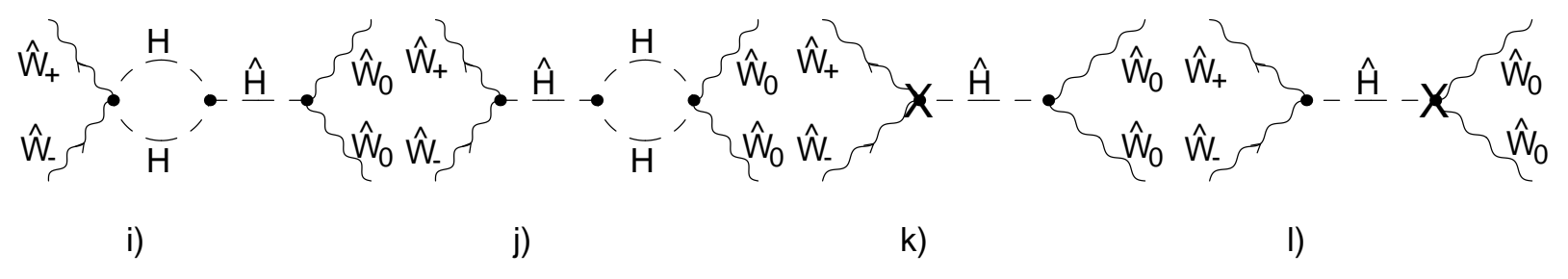

Figure 5: Reducible Higgs diagrams for the $\hat{W}^{+} \hat{W}^{-} \hat{W}^{0} \hat{W}^{0}$ four-point-function with one background Higgs field.

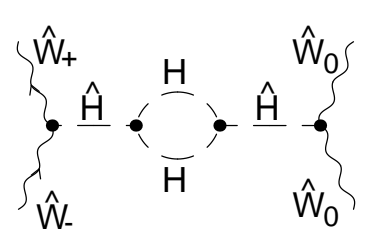

a)

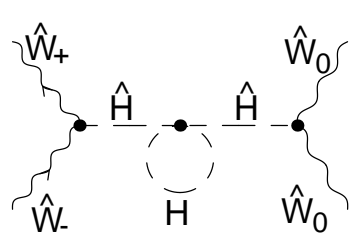

b)

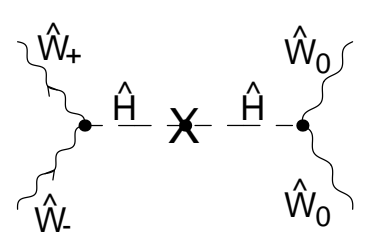

c)

Figure 6: Reducible Higgs diagrams for the $\hat{W}^{+} \hat{W}^{-} \hat{W}^{0} \hat{W}^{0}$ four-point-function with two background Higgs fields.

The diagrams with two background Higgs propagators (Fig. 6) represent the contribution of the renormalized Higgs self-energy $\Sigma^{\hat{H} \hat{H}, \text { ren }}\left(q^{2}\right)$ at $q^{2}=\left(k_{+}+k_{-}\right)^{2}$. In $\mathcal{O}\left(M_{\mathrm{H}}^{0}\right)$ one only needs $\Sigma^{\hat{H} \hat{H}, \text { ren }}(0)$ and obtains

$$
\begin{aligned}
&\left.\delta \Gamma_{\mu \nu \rho \sigma}^{\hat{W}^{+} \hat{W}^{-} \hat{W}^{0} \hat{W}^{0}}\left(k_{+}, k_{-}, k_{0,1}, k_{0,2}\right)\right|_{\hat{H} \hat{H}} \\
& \quad=\frac{i g^{4}}{16 \pi^{2}} g_{\mu \nu} g_{\rho \sigma}\left[\frac{9}{8} I_{020}-\frac{9}{8} B_{0}\left(M_{\mathrm{H}}^{2}, M_{\mathrm{H}}, M_{\mathrm{H}}\right)-\frac{3}{8} \operatorname{Re}\left\{B_{0}\left(M_{\mathrm{H}}^{2}, 0,0\right)\right\}\right],
\end{aligned}
$$

where the Higgs-mass counterterm can be read from (6.6). Of course, diagram 6b) drops out after the Higgs-mass renormalization, because its loop is scale-independent. 
Summing up all contributions to $\delta \Gamma_{\mu \nu \rho \sigma}^{\hat{W}^{+} \hat{W}^{-} \hat{W}^{0} \hat{W}^{0}}$ in $\mathcal{O}\left(M_{\mathrm{H}}^{0}\right)$, which are given in (8.9), (8.10), (8.11), we find the final result

$$
\begin{aligned}
& \delta \Gamma_{\mu \nu \rho \sigma}^{\hat{W}^{+} \hat{W}^{-} \hat{W}^{0} \hat{W}^{0}}\left(k_{+}, k_{-}, k_{0,1}, k_{0,2}\right) \\
& =\frac{i g^{4}}{16 \pi^{2}} g_{\mu \nu} g_{\rho \sigma}\left[2 I_{121}(\xi)+\frac{1}{2} I_{020}+2 I_{222}(\xi)+\frac{1}{M_{\mathrm{H}}^{2}}\left(\frac{1}{4} I_{010}-I_{111}(\xi)\right)\right. \\
& \left.-\frac{9}{8} B_{0}\left(M_{\mathrm{H}}^{2}, M_{\mathrm{H}}, M_{\mathrm{H}}\right)-\frac{3}{8} \operatorname{Re}\left\{B_{0}\left(M_{\mathrm{H}}^{2}, 0,0\right)\right\}\right] \\
& +\frac{i g^{4}}{16 \pi^{2}}\left(g_{\mu \rho} g_{\nu \sigma}+g_{\mu \sigma} g_{\nu \rho}\right) 2 I_{222}(\xi) \text {. }
\end{aligned}
$$

This is again in agreement with the result derived from the effective Lagrangian (7.6). The terms in (7.6) relevant for the Feynman rules are given by

$$
\begin{aligned}
&\left.\operatorname{tr}\left\{\hat{W}_{\mu \nu} \hat{W}^{\mu \nu}\right\}\right|_{\hat{W}^{+} \hat{W}^{-} \hat{W}^{0} \hat{W}^{0}}=2 g^{2}\left(\hat{W}^{+} \hat{W}^{-}\right)\left(\hat{W}^{0}\right)^{2}-2 g^{2}\left(\hat{W}^{+} \hat{W}^{0}\right)\left(\hat{W}^{-} \hat{W}^{0}\right), \\
&\left.\operatorname{tr}\left\{\hat{W}_{\mu \nu}\left[\hat{V}^{\mu}, \hat{V}^{\nu}\right]\right\}\right|_{\hat{W}^{+} \hat{W}^{-} \hat{W}^{0} \hat{W}^{0}}=-2 i g^{3}\left(\hat{W}^{+} \hat{W}^{-}\right)\left(\hat{W}^{0}\right)^{2}+2 i g^{3}\left(\hat{W}^{+} \hat{W}^{0}\right)\left(\hat{W}^{-} \hat{W}^{0}\right), \\
&\left.\operatorname{tr}\left\{\hat{V}_{\mu} \hat{V}_{\nu}\right\} \operatorname{tr}\left\{\hat{V}^{\mu} \hat{V}^{\nu}\right\}\right|_{\hat{W}^{+} \hat{W}^{-} \hat{W}^{0} \hat{W}^{0}}=g^{4}\left(\hat{W}^{+} \hat{W}^{0}\right)\left(\hat{W}^{-} \hat{W}^{0}\right), \\
&\left.\left(\operatorname{tr}\left\{\hat{V}_{\mu} \hat{V}^{\mu}\right\}\right)^{2}\right|_{\hat{W}^{+} \hat{W}^{-} \hat{W}^{0} \hat{W}^{0}}=g^{4}\left(\hat{W}^{+} \hat{W}^{-}\right)\left(\hat{W}^{0}\right)^{2} .
\end{aligned}
$$

The given examples clearly demonstrate the advantages of the effective Lagrangian approach for calculating the heavy-Higgs effects for specific vertex functions of the GLSM. Instead of evaluating numerous one-loop diagrams and expanding them for $M_{\mathrm{H}} \rightarrow \infty$, one can simply read the corresponding contributions directly from the effective Lagrangian. In particular, it frequently happens that several types of Feynman graphs cancel in the heavy-Higgs limit, as can be e.g. seen in the previous example of the four-point function. Such contributions do not occur in the effective Lagrangian at all.

\section{Discussion of the result}

We find that the limit $M_{\mathrm{H}} \rightarrow \infty$ of the gauged linear $\sigma$-model at one loop is the gauged non-linear $\sigma$-model plus the effective interaction terms given in (7.11). Let us emphasize that $\mathcal{L}_{\text {eff }}^{\text {ren }}(7.11)$ does not contain the complete one-loop contributions of the GLSM at $\mathcal{O}\left(M_{\mathrm{H}}^{0}\right)$, but only those effects that come from diagrams with Higgs lines. In order to find the complete one-loop corrections to an S-matrix element within the GLSM with a heavy Higgs boson, the contribution from the GNLSM Lagrangian $\mathcal{L}_{\text {GNLSM }}^{1-\text { loop }}$ in 7.8 , which still contains the light quantum fields, also has to be considered.

Since the GLSM is renormalizable, all one-loop contributions to S-Matrix elements within this model are UV-finite. In fact, the logartihmic divergencies $\Delta$ (see (A.3)) in (7.11) cancel against the logarithmically divergent contributions of the (non-renormalizable) one-loop GNLSM Lagrangian $\mathcal{L}_{\text {GNLSM }}^{1-\text { loop }}$ in 7.8 , which have been calculated in Ref. [14. 
In (7.11), logarithmically divergent contributions and $\log M_{\mathrm{H}}$-terms always occur in the linear combination $\Delta_{M_{\mathrm{H}}}$ (A.3). This and the above reasoning imply that the logarithmically divergent one-loop contributions of the GNLSM to S-matrix elements coincide with the logarithmically $M_{\mathrm{H}}$-dependent one-loop contributions in the GLSM, if one replaces

$$
\Delta=\frac{2}{4-D}-\gamma_{E}+\log (4 \pi)+\log \mu^{2} \rightarrow \log M_{\mathrm{H}}^{2},
$$

as assumed in Ref. [14; i.e. the $\log M_{\mathrm{H}^{-}}$effects of the GLSM can alternatively be calculated within the GNLSM. However, the Lagrangian (7.11) contains additional finite and $M_{\mathrm{H}^{-}}$ independent contributions, which describe differences between the GLSM and the GNLSM at one loop. Thus, the GNLSM with the replacement (9.1) is not the heavy-Higgs limit of the GLSM at one loop, but it differs from this by finite, constant contributions. Since the logarithm increases very slowly, these constants are even for a large Higgs mass of a magnitude comparable to that of the $\log M_{\mathrm{H}}$-terms, and thus they have to be taken into account.

Finally, we compare our result with that found in Ref. [1] by diagrammatical calculations for the electroweak standard model, which can be reduced to the $\mathrm{SU}(2)$ model by setting $g^{\prime}=0$ there. We find that our result (7.11) agrees with that of Ref. [1]. However, it should be noted, that we find a different factor for the $\operatorname{tr}\left\{\left(\hat{D}_{W}^{\mu} \hat{V}_{\mu}\right)^{2}\right\}$-term in $(7.7)$. The reason for this is that in Ref. [1] the linear parametrization of the scalar sector (2.3) is used, while we applied the non-linear parametrization (2.5), (2.6). It is well-known that such a reparametrization of the scalar fields leaves S-matrix elements unaffected, however, it may change Green functions [16]. As pointed out in the previous section, the $\operatorname{tr}\left\{\left(\hat{D}_{W}^{\mu} \hat{V}_{\mu}\right)^{2}\right\}$-term in (7.7) has no effect on S-matrix elements. Thus, our result and that of Ref. [1] are consistent with each other.

\section{Summary}

In this article we have described a general method to remove non-decoupling heavy fields from a quantized field theory at one loop and to construct a low-energy one-loop effective Lagrangian by functional methods, i.e. by integrating out the heavy degrees of freedom in the path integral. We have applied this method to a specific example, viz. a spontaneously broken $\mathrm{SU}(2)$ gauge theory, but it can immediately be applied to any other model with a non-decoupling heavy field in order to construct its $M \rightarrow \infty$ limit at one loop, where $M$ is the mass of the heavy field.

We have used the background-field method, where the fields are split into classical background fields, which correspond to tree lines, and quantum fields, which correspond to lines inside loops. The heavy quantum field is integrated out by performing the integration over this degree of freedom in the path integral, while the corresponding background field can then be removed by a propagator expansion of its tree lines in $1 / M$ or by applying the classical equations of motion in lowest order. The resulting Lagrangian still contains the light quantum fields, i.e. it does not parametrize the complete one-loop effects of the theory but only the contribution from loops with heavy particles. However, the effective terms generated by integrating out the heavy field contain only background fields, because 
these terms already parametrize one-loop effects, and thus, only have to be used at tree level, when subsequently calculating vertex functions or S-matrix elements at one loop.

Comparing our functional approach with diagrammatical calculations (see Ref. [1] and Sect. 8), we find that it possesses many advantages: The $1 / M$-expansion described in Sect. 1, i.e. the isolation of the non-decoupling effects of the heavy fields from the decoupling effects, is very easy within the functional approach. Furthermore, our calculations could be done within the convenient matrix notation; i.e. we had not to write down the components of the fields. This property and the application of the Stueckelberg formalism, which removes the background Goldstone fields from intermediate calculations, enables the simultaneous calculation of one-loop contributions to many different Green functions. For instance, our final result also contains contributions to Green functions with external Goldstone fields, although these fields never occurred explicitly during our calculation. The use of the matrix notation and of the Stueckelberg formalism also made it very easy to write the generated effective Lagrangian into a manifestly gauge-invariant form.

We have applied this method to integrate out the Higgs boson in the $\mathrm{SU}(2)$ gauged linear $\sigma$-model at one loop. We have found that the logarithmically $M_{\mathrm{H}^{-}}$dependent contributions to S-matrix elements within this model coincide with the logarithmically divergent contributions of the gauged non-linear $\sigma$-model if the substitution (9.1) is done, however that the latter model differs from the heavy-Higgs limit of the former by finite and constant contributions at one loop.

As a by-product of this calculation we have formulated the background-field method for spontaneously broken gauge theories for the case that the scalar sector is non-linearly parametrized, and we have generalized the Stueckelberg formalism to the backgroundfield method. The renormalization has been carried out such that also the renormalized effective action remains background-gauge-invariant.

We will apply the method described in this article to integrate out the (heavy) Higgs boson in the electroweak standard model in a forthcoming article [12].

\section{Acknowledgement}

We thank Ansgar Denner and Reinhart Kögerler for helpful discussions and for reading the manuscript.

\section{Appendix}

\section{A Explicit expressions for the one-loop integrals}

In Sect. 1 the construction of the unrenormalized effective Lagrangian (4.27) was traced back to the vacuum integrals $I_{k l m}(\xi)$ defined in (4.26). Such vacuum integrals are easily calculated to

$$
\frac{(2 \pi \mu)^{4-D}}{i \pi^{2}} \int d^{D} p \frac{p_{\mu_{1}} \ldots p_{\mu_{2 k}}}{\left(p^{2}-M_{1}^{2}\right)^{l}}=g_{\mu_{1} \ldots \mu_{2 k}} \frac{(-1)^{k+l}}{2^{k}} \frac{\Gamma\left(l-k-\frac{D}{2}\right)}{\Gamma(l)}\left(4 \pi \mu^{2}\right)^{\frac{4-D}{2}} M^{D+2 k-2 l},
$$




$$
\begin{gathered}
\frac{(2 \pi \mu)^{4-D}}{i \pi^{2}} \int d^{D} p \frac{p_{\mu_{1}} \ldots p_{\mu_{2 k}}}{\left(p^{2}-M_{1}^{2}\right)^{l}\left(p^{2}-M_{2}^{2}\right)^{m}}=g_{\mu_{1} \ldots \mu_{2 k}} \frac{(-1)^{k}}{2^{k}} \frac{\Gamma\left(1-k-\frac{D}{2}\right)}{\Gamma(l) \Gamma(m)}\left(4 \pi \mu^{2}\right)^{\frac{4-D}{2}} \\
\times\left(\frac{\partial}{\partial M_{1}^{2}}\right)^{l-1}\left(\frac{\partial}{\partial M_{2}^{2}}\right)^{m-1}\left[\frac{M_{1}^{D+2 k-2}-M_{2}^{D+2 k-2}}{M_{2}^{2}-M_{1}^{2}}\right],
\end{gathered}
$$

for arbitrary space-time dimension $D$. According to (A.1) the relevant $\mathcal{O}\left(M_{\mathrm{H}}^{0}\right)$ parts of the $I_{k l m}$ for $D \rightarrow 4$ are given by

$$
\begin{aligned}
I_{010} & =M_{\mathrm{H}}^{2}\left(\Delta_{M_{\mathrm{H}}}+1\right), \\
I_{011}(\xi) & =\Delta_{M_{\mathrm{H}}}+1+\mathcal{O}\left(M_{\mathrm{H}}^{-2}\right), \\
I_{020} & =\Delta_{M_{\mathrm{H}}} \\
I_{111}(\xi) & =\frac{1}{4}\left(M_{\mathrm{H}}^{2}+\xi M_{\mathrm{W}}^{2}\right)\left(\Delta_{M_{\mathrm{H}}}+\frac{3}{2}\right)+\mathcal{O}\left(M_{\mathrm{H}}^{-2}\right), \\
I_{112}(\xi) & =\frac{1}{4}\left(\Delta_{M_{\mathrm{H}}}+\frac{3}{2}\right)+\mathcal{O}\left(M_{\mathrm{H}}^{-2}\right), \\
I_{121}(\xi) & =\frac{1}{4}\left(\Delta_{M_{\mathrm{H}}}+\frac{1}{2}\right)+\mathcal{O}\left(M_{\mathrm{H}}^{-2}\right), \\
I_{213}(\xi) & =\frac{1}{24}\left(\Delta_{M_{\mathrm{H}}}+\frac{11}{6}\right)+\mathcal{O}\left(M_{\mathrm{H}}^{-2}\right), \\
I_{222}(\xi) & =\frac{1}{24}\left(\Delta_{M_{\mathrm{H}}}+\frac{5}{6}\right)+\mathcal{O}\left(M_{\mathrm{H}}^{-2}\right),
\end{aligned}
$$

with

$$
\Delta_{M_{\mathrm{H}}}=\Delta-\log \left(\frac{M_{\mathrm{H}}^{2}}{\mu^{2}}\right), \quad \Delta=\frac{2}{4-D}-\gamma_{E}+\log (4 \pi),
$$

and $\gamma_{E}$ being Euler's constant.

In Sect. 6 we expressed the renormalization constant $\delta M_{\mathrm{H}}^{2}$ (6.7) in terms of $I_{k l m}$ and scalar two-point functions $B_{0}\left(k^{2}, M_{1}, M_{2}\right)$ defined in (6.8). The explicit expressions for the relevant $B_{0}$-functions can for instance be deduced from the general result presented in Ref. [18, leading to

$$
\begin{aligned}
B_{0}\left(M_{\mathrm{H}}^{2}, M_{\mathrm{H}}, M_{\mathrm{H}}\right) & =\Delta_{M_{\mathrm{H}}}+2-\frac{\pi}{\sqrt{3}}, \\
B_{0}\left(M_{\mathrm{H}}^{2}, 0,0\right) & =\Delta_{M_{\mathrm{H}}}+2+i \pi .
\end{aligned}
$$

\section{References}

[1] M.J. Herrero and E. Ruiz Morales, Nucl. Phys. B418 (1994) 431; Madrid preprint FTUAM 94/11 (1994), hep-ph/9411207

[2] M. Bilenky and A. Santamaria, Nucl. Phys. B420 (1994) 47

[3] J. Gasser and H. Leutwyler, Ann. Phys. (NY) 158 (1984) 142;

A. Nyffeler and A. Schenk, Bern Preprint BUTP-94/12 (1994), hep-ph/9409436 
[4] I.J.R. Aitchison and C.M. Fraser, Phys. Lett. B146 (1984) 63; Phys. Rev. D31 (1985) 2605 ;

C.M. Fraser, Z. Phys. C28 (1985) 101;

J.A. Zuk, Phys. Rev. D32 (1985) 2653; D33 (1986) 3545;

O. Cheyette, Phys. Rev. Lett. 55 (1985) 2394;

M.K. Gaillard, Nucl. Phys. B268 (1986) 669;

L.-H. Chan, Phys. Rev. D36 (1987) 3755;

R.D. Ball, Phys. Rep. 182 (1989) 1

[5] L.-H. Chan, Phys. Rev. Lett. 54 (1985) 1222 [Err. 56 (1986) 404]; 57 (1986) 1199

[6] O. Cheyette, Nucl. Phys. B297 (1988) 183

[7] B.S. DeWitt, Phys. Rev. 162 (1967) 1195; Dynamical Theory of groups and Fields (Gordon and Breach, New York, 1965); in Quantum Gravity 2, ed. C.J. Isham, et. al. (Oxford University Press, New York, 1981), p. 449;

G. 't Hooft, Acta Universitatis Wratislavensis 368 (1976) 345;

H. Kluberg-Stern and J. Zuber, Phys. Rev. D12 (1975) 482 and 3159;

D.G. Bouleware, Phys. Rev. D23 (1981) 389;

C.F. Hart, Phys. Rev. D28 (1983) 1993

[8] L.F. Abbott, Nucl. Phys. B185 (1981) 189; Acta. Phys. Pol. B13 (1982) 33;

L.F. Abbott, M.T. Grisaru and R.K. Schaefer, Nucl. Phys. B229 (1983) 372

[9] M.B. Einhorn and J. Wudka, Phys. Rev. D39 (1989) 2758

[10] A. Denner, S. Dittmaier and G. Weiglein, Phys. Lett. B333 (1994) 420; Nucl. Phys. B (Proc. Suppl.) 37B (1994) 87

[11] A. Denner, S. Dittmaier and G. Weiglein, Bielefeld Preprint 94/50 (1994), hep$\mathrm{ph} / 9410338$

[12] S. Dittmaier and C. Grosse-Knetter, in preparation

[13] W.A. Bardeen and K. Shizuya, Phys. Rev. D18 (1978) 1969

[14] T. Appelquist and C. Bernard, Phys. Rev. D22 (1980) 200;

A. C. Longhitano, Nucl. Phys. B188 (1981) 118

[15] E. C. G. Stueckelberg, Helv. Phys. Acta 11 (1938) 299; 30 (1956) 209;

T. Kunimasa and T. Goto, Prog. Theor. Phys. 37 (1967) 425

[16] B. W. Lee and J. Zinn-Justin, Phys. Rev. D5 (1972) 3155;

C. Grosse-Knetter and R. Kögerler, Phys. Rev. D48 (1993) 2865

[17] K.I. Aoki, Z. Hioki, R. Kawabe, M. Konuma and T. Muta, Prog. Theor. Phys. 64 (1980) 707; 65 (1981) 1001; Suppl. Prog. Theor. Phys. 73 (1982) 1;

M. Böhm, W. Hollik and H. Spiesberger, Fortschr. Phys. 34 (1986) 687

[18] A. Denner, Fortschr. Phys. 41 (1993) 307 
[19] D. Barua and S. N. Gupta, Phys. Rev. D16 (1977) 413;

H. D. Politzer, Nucl. Phys. B172 (1980) 349;

H. Georgi, Nucl. Phys. B361 (1991) 339;

C. Arzt, Michigan-Preprint UM-TH-92-28 (1992), hep-ph/9304230;

H. Simma, Z. Phys. C61 (1994) 67;

C. Grosse-Knetter, Phys. Rev. D49 (1994) 1988 and 6709 